\title{
Geochemistry and statistical analyses of porphyry system and epithermal veins at Hizehjan in north- western Iran
}

\author{
Kaikhosrov Radmard ${ }^{1, *}$, Hassan Zamanian ${ }^{1}$, Mohamad Reza Hosseinzadeh ${ }^{2}$, \\ Ahmad Ahmadi Khalaji ${ }^{1}$
}

\begin{abstract}
${ }^{1}$ Department of Geology, Faculty of Natural Sciences, Lorestan University, Khoram Abad, Iran ${ }^{2}$ Department of Earth Sciences, Faculty of Natural Sciences, University of Tabriz, Tabriz, Iran *corresponding author, e-mail: k_radmard@yahoo.com
\end{abstract}

\begin{abstract}
Situated about $130 \mathrm{~km}$ northeast of Tabriz (northwest Iran), the Mazra'eh Shadi deposit is in the Arasbaran metallogenic belt (AAB). Intrusion of subvolcanic rocks, such as quartz monzodiorite-diorite porphyry, into Eocene volcanic and volcano-sedimentary units led to mineralisation and alteration. Mineralisation can be subdivided into a porphyry system and Au-bearing quartz veins within andesite and trachyandesite which is controlled by fault distribution. Rock samples from quartz veins show maximum values of $\mathrm{Au}(17100 \mathrm{ppb}), \mathrm{Pb}(21100 \mathrm{ppm}), \mathrm{Ag}(9.43 \mathrm{ppm}), \mathrm{Cu}(611 \mathrm{ppm})$ and $\mathrm{Zn}(333 \mathrm{ppm}) . \mathrm{Au}$ is strongly correlated with $\mathrm{Ag}$, $\mathrm{Zn}$ and $\mathrm{Pb}$. In the Au-bearing quartz veins, factor group 1 indicates a strong correlation between $\mathrm{Au}, \mathrm{Pb}, \mathrm{Ag}, \mathrm{Zn}$ and $\mathrm{W}$. Factor group 2 indicates a correlation between $\mathrm{Cu}, \mathrm{Te}, \mathrm{Sb}$ and $\mathrm{Zn}$, while factor group 3 comprises Mo and As. Based on Spearman correlation coefficients, Sb and Te can be very good indicator minerals for $\mathrm{Au}, \mathrm{Ag}$ and $\mathrm{Pb}$ epithermal mineralisation in the study area. The zoning pattern shows clearly that base metals, such as $\mathrm{Cu}, \mathrm{Pb}, \mathrm{Zn}$ and Mo, occur at the deepest levels, whereas $\mathrm{Au}$ and Ag are found at higher elevations than base metals in boreholes in northern Mazra'eh Shadi. This observation contrasts with the typical zoning pattern caused by boiling in epithermal veins. At Mazra'eh Shadi, quartz veins containing co-existing liquid-rich and vapour-rich inclusions, as strong evidence of boiling during hydrothermal evolution, have relatively high Au grades (up to $813 \mathrm{ppb}$ ). In the quartz veins, $\mathrm{Au}$ is strongly correlated with $\mathrm{Ag}$, and these elements are in the same group with $\mathrm{Fe}$ and S. Mineralisation of $\mathrm{Au}$ and $\mathrm{Ag}$ is a result of pyrite precipitation, boiling of hydrothermal fluids and a $\mathrm{pH}$ decrease.
\end{abstract}

Key words: Mazra'eh Shadi-Hizehjan, quartz monzodiorite, Spearman correlation, boiling

\section{Introduction}

One of the most fundamental aims in geochemical studies is to elucidate significant anomalies, determine their relationships, use correlation analyses and assess mineralisation zones so as to determine the type of exploration effectively. Most intermediate-sulfidation epithermal $\mathrm{Au}$ and $\mathrm{Ag}$ deposits are found in magmatic arcs, in calcalkaline andesitic-dacitic and more felsic rocks. A few intermediate- sulfidation deposits, typically small ones, adjoin advanced argillic lithocaps, with or without associated high- sulfidation epithermal deposits (Sillitoe, 1999). The Mazra' eh Shadi deposit is located about $130 \mathrm{~km}$ northeast of Tabriz (northwest Iran) in the Arasbaran metallogenic belt. Based on primary studies, such as field survey, petrographical and mineralogical investigation, the Mazra'eh Shadi region is an appropriate $\mathrm{Cu}$ and $\mathrm{Au}$ resource. Based upon a classification of structural domains of Iran (Nabavi, 1976), 


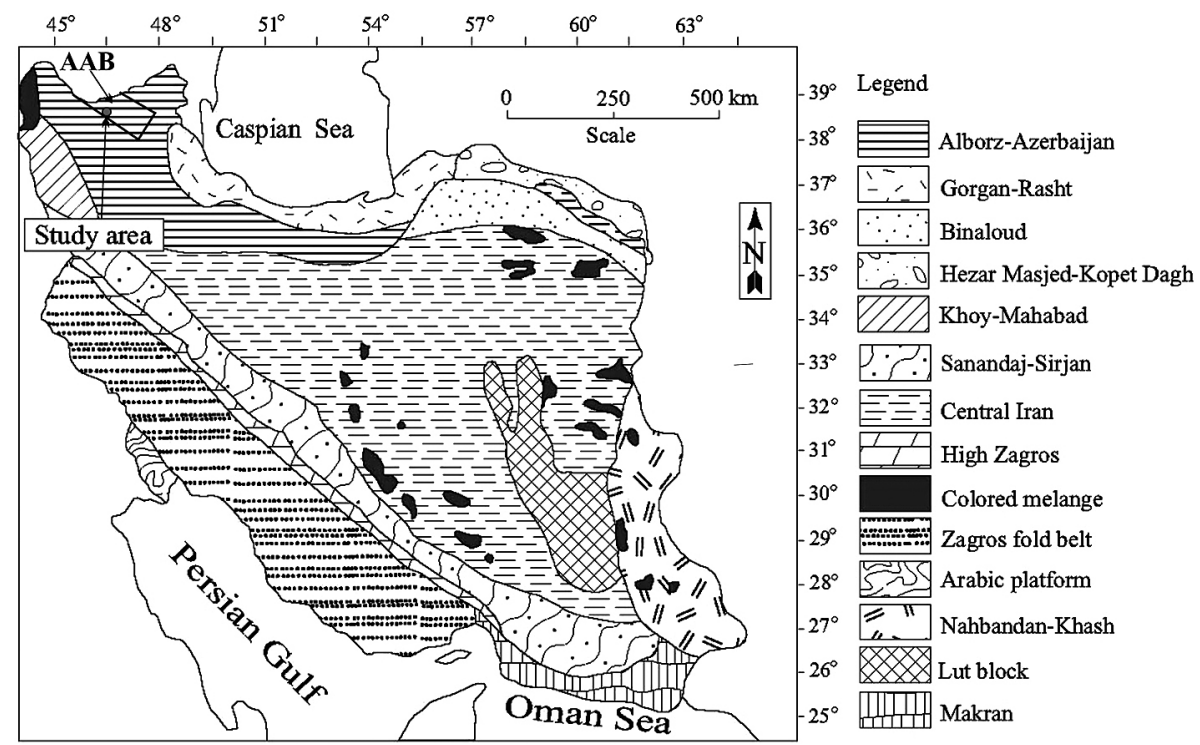

Fig. 1. Simplified regional geotectonic map of Iran (Nabavi, 1976) showing major geological-structural zones. AAB Arasbaran metallogenic belt.

the area is part of the Alborz-Azerbaijan Zone (Fig. 1 ) and is located in the Ahar-Arasbaran Belt (AAB) which is characterised by a high potential of precious and base metal mineralisations. More than 10 $\mathrm{Cu} \pm \mathrm{Mo} \pm \mathrm{Au}$ porphyry deposits and epithermal gold systems have been recorded from the Arasbaran metallogenic zone (e.g., at Songon, Mazraea, Masjed Dagy, Zegleg and Darian). The purpose of the present paper is to discuss geological and geochemical data that can be used to (1) determine elemental zoning in the Arasbaran metallogenic belt, (2) provide an exploration model for epithermal deposits with characteristics similar to those of the Arasbaran metallogenic belt (3) enhance our current understanding of the processes involved in forming Au epithermal deposits. The main structure in the Arasbaran metallogenic region formed as a result of Alpine orogeny, especially of the Late Cretaceous Laramide phase.

\section{Material and methods}

The recent discovery of epithermal and porphyry style mineralisations in the Mazra'eh Shadi-Hizehjan region suggests that the area could potentially be productive and is in need of further investigations.

Based on previous studies more than 400 samples have been collected from surface and drill core samples in the Mazra'eh Shadi-Hizehjan region. Forty five thin and polished sections were studied by optical microscopy at Tabriz University. For determination of major rock-forming minerals in the ores, 63 samples were selected for X-ray diffraction (XRD) analysis using a SIEMENS model D-5000 diffractometer with $\mathrm{CuKa}$ radiation, voltage $40 \mathrm{kV}$, beam current $80 \mathrm{~mA}$, continuous scanning, scanning speed $8^{\circ} / \mathrm{min}$, scan range $2^{\circ}-70^{\circ}$, slit DS $=$ SS $=1^{\circ}$, ambient temperature $18^{\circ} \mathrm{C}$ and humidity $30 \%$ in Binalod laboratory (Iran). Rock samples were crushed to 200-mesh size particles using an agate mill. The chemical analyses were performed in the Amdel laboratory (Australia) and ALS Chemex Canada. Gold was analysed by inductively coupled plasma optical emission spectrometry (ICP-OES) and $\mathrm{Ag}, \mathrm{Pb}, \mathrm{Zn}, \mathrm{Cu}, \mathrm{As}, \mathrm{Sb}$ by inductively coupled plasma mass spectrometry (ICP-MS). Detection limits for these elements were: $\mathrm{Au}=1 \mathrm{ppb}, \mathrm{Ag}=0.2 \mathrm{ppm}$, $\mathrm{Pb}, \mathrm{Zn}$ and $\mathrm{Cu}=0.5$ ppm (Tables 1-2). Ten samples containing quartz veinlets from diamond drill holes and surface samples were selected for thermometric analyses. Microthermometric studies were conducted on 100-150- $\mu$ m-thick polished slabs prepared from ten quartz samples from silicified and mineralised zones. Most samples were from goldrich and quartz-sulphide breccia veins. Ten subsurface samples containing quartz veinlets from diamond drill holes and surface samples were selected for thermometric analyses. Microthermometric studies were carried out at the Lorestan University, Geological Department-Fluid Inclusion Laboratory, using a Linkam THMSG600 freezing-heating stage mounted on an Olympus microscope. Freezing-heating stage has $\pm 0.1^{\circ} \mathrm{C}$ precision and the temperature range varies from -196 to $600{ }^{\circ} \mathrm{C}$. In order to prevent fluid inclusions from cracking during heating, a cooling process has to be employed. Salinities of liquid-rich fluid inclusions were calculated from measured ice-melting temperatures using 
Table 1. Results of chemical analyses of quartz monzodiorite. Au is in ppb; other elements are in ppm. Gold analyses were performed by fire assay, while other elements were analysed by inductively coupled plasma mass spectrometry (ICP-MS).

\begin{tabular}{|c|c|c|c|c|c|c|c|c|c|c|c|c|c|c|c|c|c|c|c|c|}
\hline & & & & & & & & & & & & & $1 \mathrm{C}$ & & N & & & & & \\
\hline & 3 & 2.6 & & & & & & & & & & 0.9 & 0.2 & & & & & & & \\
\hline & & & & & .4 & & & & & & & 7 & & & & & & & & \\
\hline & & & & & & & & & & & & & & & & & & & & \\
\hline & 2 & & 29 & & & & & & & & 0.4 & 0.4 & .5 & & & & & .3 & & \\
\hline & 2 & & & & 77 & & & & & & & & & & & & & & & \\
\hline & 73 & & & & . & & & & & & 3.4 & 3.2 & 3 & & & & & .7 & & \\
\hline & 2 & & & & 55 & & & & & & 0.7 & & & 4.7 & & & & 1.2 & & 0.4 \\
\hline & 14 & & & & & & & & & & 7 & & & & & & & & & \\
\hline & 5 & & & .4 & 1 & & & 3 & & & 1 & 0.6 & & 4.4 & & & & .5 & & \\
\hline & 3 & & & & & & & & & & & & & & & & & & & \\
\hline & 5 & & & & & & & 6 & & & 0.7 & 0.8 & 1.5 & & & & & 0.7 & & \\
\hline & & & & & & & & & & & & & & & & & & & & \\
\hline & 7 & 7 & & & & & & & & & 1 & & 4 & & & & & & & \\
\hline & 3 & & & & 76.2 & & & & & & 1.2 & & & & & & & & & \\
\hline & 31 & & & & 46 & & & & & & 2 & & . & & & & & .7 & & 0.2 \\
\hline & 2 & & & & & & & & & & 0 & & & & & & & & & \\
\hline & 16 & & & & & & & & & & & & 0.3 & 13 & & & & 6.4 & & 0 \\
\hline & & & & & & & & & & & & 1.5 & 0.2 & & & & & 5 & & \\
\hline & & & & & & & & & & & & 4 & & & & & & & & \\
\hline & 277 & & & חרת & 981 & & & & & & 0.5 & 6.7 & 0.7 & 95.6 & & & & .1 & 4 & \\
\hline & 59 & & & 143 & & & & & & & & & 1 & & & & & 0.1 & & \\
\hline & 30 & & & 44 & & 5 & & & & & 0.8 & 1.9 & 1.5 & 101 & 28200 & & 4310 & 1.2 & 12 & 0.2 \\
\hline KSI31 & 798 & 618 & 0.42 & 149 & 1650 & & 7.2 & 4.2 & 0.3 & 11800 & 0 & 0.2 & 0.2 & 16.8 & 5340 & 3400 & 142 & 0.3 & 4 & 27 \\
\hline
\end{tabular}

Table 2. Results of chemical analyses in Au-bearing quartz veins. Au is in ppb; other elements in ppm. Gold analyses were performed by fire assay, while other elements were analysed by inductively coupled plasma mass spectrometry (ICP-MS).

\begin{tabular}{lcccccccccccccccccc}
\hline & $\mathrm{Au}$ & $\mathrm{Pb}$ & $\mathrm{Ag}$ & $\mathrm{Cu}$ & $\mathrm{Zn}$ & $\mathrm{Mo}$ & $\mathrm{As}$ & $\mathrm{Sb}$ & $\mathrm{Bi}$ & $\mathrm{S}$ & $\mathrm{Sn}$ & $\mathrm{W}$ & $\mathrm{Te}$ & $\mathrm{Rb}$ & $\mathrm{K}$ & $\mathrm{Mn}$ & $\mathrm{Ti}$ & $\mathrm{Tl}$ \\
\hline KSI56 & 8 & 8.3 & 0.36 & 30.3 & 5.9 & 6.3 & 684 & 0.9 & 0.1 & 21100 & 0.9 & 1.9 & 0 & 5.1 & 6840 & 62 & 3560 & 0.3 \\
KSI57 & 2 & 11 & 0.23 & 15 & 2.4 & 8.5 & 377 & 0.7 & 0.2 & 12200 & 0.9 & 1.5 & 0 & 2.9 & 4650 & 80 & 3030 & 0 \\
\hline KSI59 & 2 & 11.4 & 0.28 & 23.1 & 3.3 & 4.3 & 382 & 0.7 & 0.1 & 16400 & 1.2 & 1.7 & 0 & 3.3 & 4040 & 65 & 4280 & 0.9 \\
KSI60 & 1 & 12.2 & 0.2 & 32.2 & 2.4 & 3.3 & 278 & 0.6 & 0.1 & 38600 & 0.8 & 1.4 & 0 & 4.4 & 13100 & 78 & 3660 & 0.4 \\
KSI65 & 9 & 8 & 0.23 & 13.4 & 0.9 & 5.9 & 53 & 0.8 & 0.1 & 2250 & 1.1 & 1.3 & 0 & 1.7 & 1260 & 45 & 3840 & 0.1 \\
KSI66 & 3 & 13.2 & 0.39 & 42.2 & 5.8 & 9 & 145 & 1 & 0.1 & 4430 & 1.6 & 1.6 & 0 & 2 & 1830 & 76 & 6650 & 1.4 \\
\hline KSI71 & 4 & 17.5 & 0.36 & 261 & 14.7 & 18 & 1820 & 1 & 0.2 & 5750 & 1 & 1.3 & 0 & 1.5 & 1480 & 127 & 4610 & 0.3 \\
KSI73 & 3 & 24.8 & 0.32 & 33.3 & 3.2 & 8.5 & 151 & 1.3 & 0 & 20700 & 1.2 & 2.9 & 0 & 3.3 & 5990 & 88 & 5750 & 0.6 \\
\hline KSI74 & 5 & 32.5 & 0.23 & 16.7 & 3.7 & 9.2 & 93.1 & 1.1 & 0.6 & 18600 & 1 & 3.4 & 0 & 2.1 & 5440 & 33 & 4040 & 0 \\
\hline KSI75 & 895 & 4300 & 3.21 & 611 & 144 & 9 & 85.4 & 37.7 & 0.2 & 20100 & 0.5 & 2.1 & 1.3 & 9.1 & 8300 & 65 & 848 & 0.4 \\
KSI77 & 456 & 3120 & 6.1 & 158 & 71.5 & 4.9 & 249 & 13.1 & 0.9 & 9630 & 0.6 & 3.2 & 0.9 & 5.5 & 4690 & 124 & 1880 & 0.5 \\
KSI78 & 2420 & 3840 & 1.69 & 68.9 & 333 & 12.3 & 699 & 25.4 & 0.2 & 20800 & 0.6 & 6.8 & 0.4 & 25.6 & 8580 & 71 & 3110 & 7.3 \\
\hline KSI81 & 17100 & 21100 & 9.43 & 251 & 182 & 1.8 & 114 & 15.6 & 0.2 & 16500 & 0.5 & 4.2 & 0.6 & 4.1 & 5790 & 102 & 498 & 0.7 \\
KSI82 & 122 & 153 & 0.87 & 8.2 & 10.4 & 2.1 & 28.6 & 1.7 & 0.6 & 18300 & 0.8 & 1.1 & 0.8 & 76.5 & 31300 & 28 & 2120 & 1.6 \\
\hline KSI85 & 29 & 66.6 & 0.73 & 45 & 7.1 & 3.1 & 17.2 & 1.1 & 0.8 & 2200 & 2 & 1.3 & 0.5 & 97.3 & 33200 & 61 & 3610 & 1.7 \\
KSI87 & 1280 & 867 & 2.94 & 13.9 & 31.8 & 2 & 11.6 & 28.5 & 0.6 & 1180 & 0.6 & 0.2 & 0 & 41 & 12200 & 49 & 740 & 0.6 \\
\hline
\end{tabular}

the equation of Bodnar (1993). Interpolation of data and creation of longitudinal sections for the veins was done using the Kriging interpolation function built in the Surfer software, version 9, Excel, Auto CAD. The basic statistics, minimum, maximum, mean and standard deviation were calculated for every vein by SPSS software, version 16.

\section{Geology}

In the study area three types of lithologies are represented (Radmard et al., 2017; Fig. 2), as follows:

1. Eocene pyroclastic rocks, consisting especially of tuff and lava usually of an andesitic composition; 


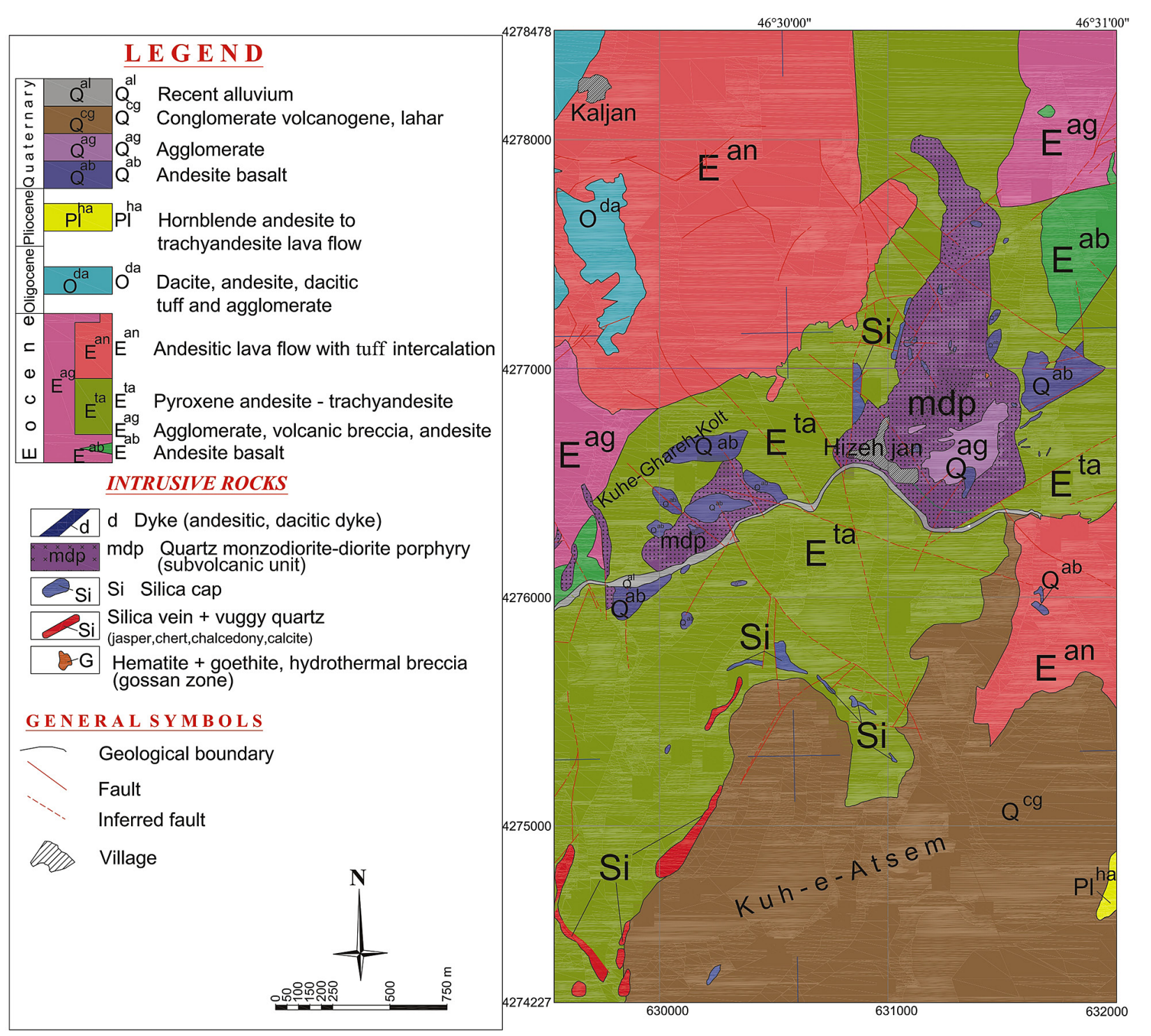

Fig. 2. Geological map of the Mazra'eh Shadi-Heizehjan region (Radmard et al., 2017).

2. Oligocene-Pliocene rocks, consisting of hornblende andesite to trachyandesite lava, dacite tuff and agglomerate;

3. Quaternary volcanogenic conglomerate (agglomerate) and alluvial units.

The Eocene pyroclastic, volcanic and agglomerate (usually of an andesitic composition) are covered by dacitic tuff, dacite, hornblende andesite, trachyandesite and andesite volcanic rocks of Oligocene and Pliocene age. Numerous dacite to andesitic dykes intruded into volcanic and pyroclastic rocks of Late Eocene age. The youngest rock units in the area are Quaternary alluvial plain and river sediments. The Quaternary volcanogenic conglomerate (agglomerate) crops out in the vicinity of the Mazra'eh Shadi deposit in the southwestern part of the study area (Fig. 2). Subvolcanic rocks such as quartz monzodiorite-diorite porphyry intruded into
Eocene volcanic and volcano-sedimentary units and caused mineralisation and alteration. Gold mineralisation in the Hizehjan area is related to Eocene volcanic and intrusive rocks. Outcrops of subvolcanic rocks extend over a distance of about 1,650 $\mathrm{m}$ and their width ranges from 300 to $450 \mathrm{~m}$ in the area.

\section{Results}

\subsection{Alteration}

Hydrothermal alteration zones are well developed around the Mazra'eh Shadi veins and Hizehjan. Hydrothermal activity and the presence of faults and highly fractured breccia zones led to a range of alteration phenomena in Mazra'eh Shadi (Radmard et al., 2017; Fig. 3). Alteration associated with 


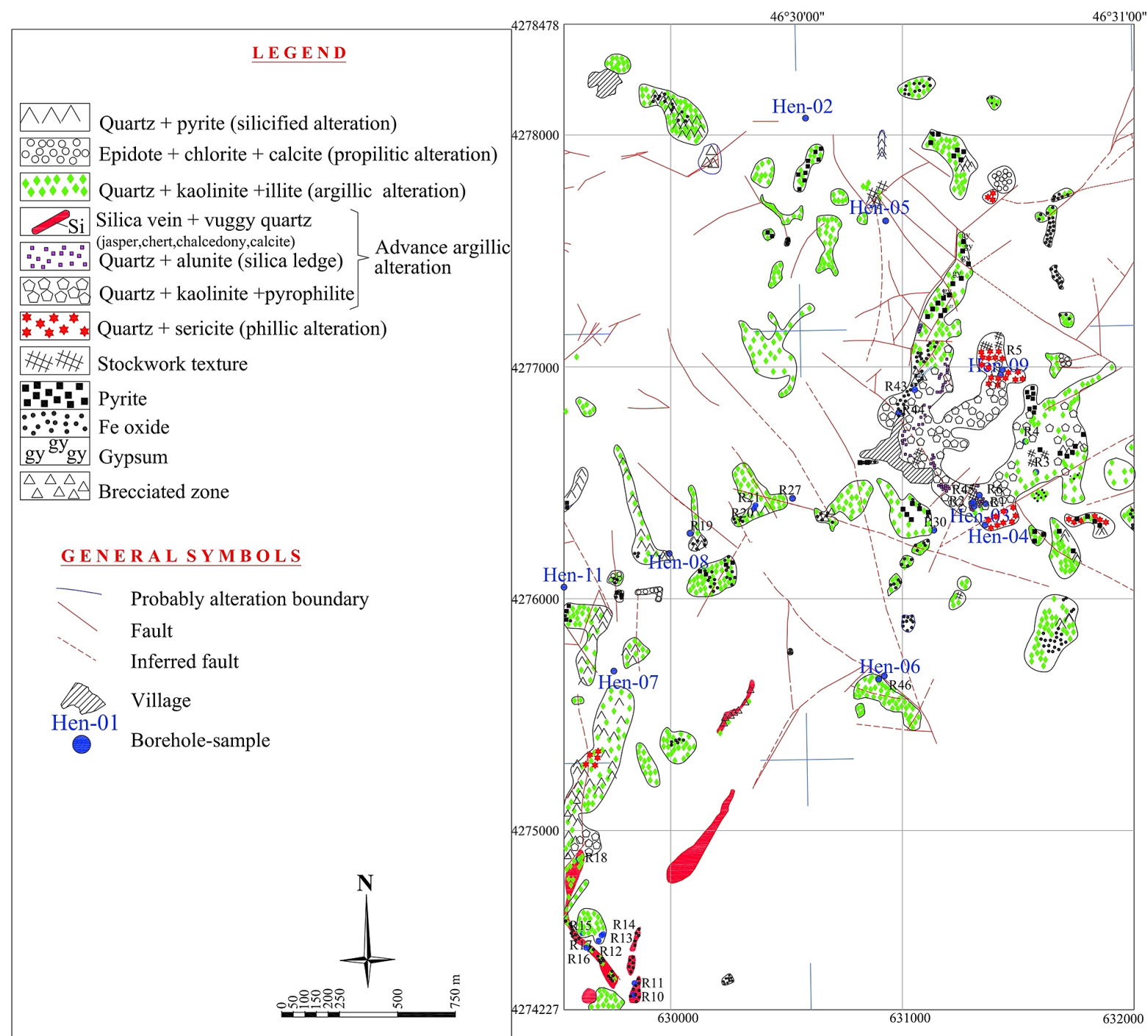

Fig. 3. Map of alteration zones in the Mazra'eh Shadi-Heizehjan region (Radmard et al., 2017).

mineralisation is confined to thin halos of silicified rocks adjacent to the veins, bordered by argillic and propylitic zones outwards. Field mapping, microscopic study and XRD analysis (Table 3) have resulted in the recognition of five, zonally distributed hydrothermal alteration assemblages that consist of propylitisation, phyllic, argillic-advanced argillic alterations and potassic associated with silicification and ore deposition.

Generally, outcrops of silicification can be divided into three main forms: stockwork in the north- eastern part of the area; distinct silica caps at outcrops in the central and southwestern parts and quartz veins with an $\mathrm{N} 20-80^{\circ} \mathrm{E}$ trend in the southwestern part. The presence of several scattered and separated silica caps in argillic alteration indicates that the surface of silica caps are rough, highlighting this morphology due to their resistance to weathering (Fig. 4A).

The argillic alteration is subdivided into argillic and advanced argillic alterations. Argillic alteration is present in the central part of the area; it led to

Table 3. Minerals (identified by XRD analyses) in hydrothermal alteration zones of the Mazra' eh Shadi deposit.

\begin{tabular}{lll}
\hline \multicolumn{1}{c}{ Alteration zones } & \multicolumn{1}{c}{ Major Minerals } & \multicolumn{1}{c}{ Minor Minerals } \\
\hline Propylitic & chlorite, albite, calcite, & montmorillonite, calcite \\
Phyllic & quartz, muscovite, illite, & chlorite, kaolinite, natrojarosite, jarosite, pyrite \\
Argillic & quartz, kaolinite, montmorillonite & muscovite, calcite, goethite, illite, chlorite, \\
Advanced argillic & kaolinite, quartz, gypsum, & muscovite, illite, alunite, montmorillonite, hematite \\
\hline
\end{tabular}



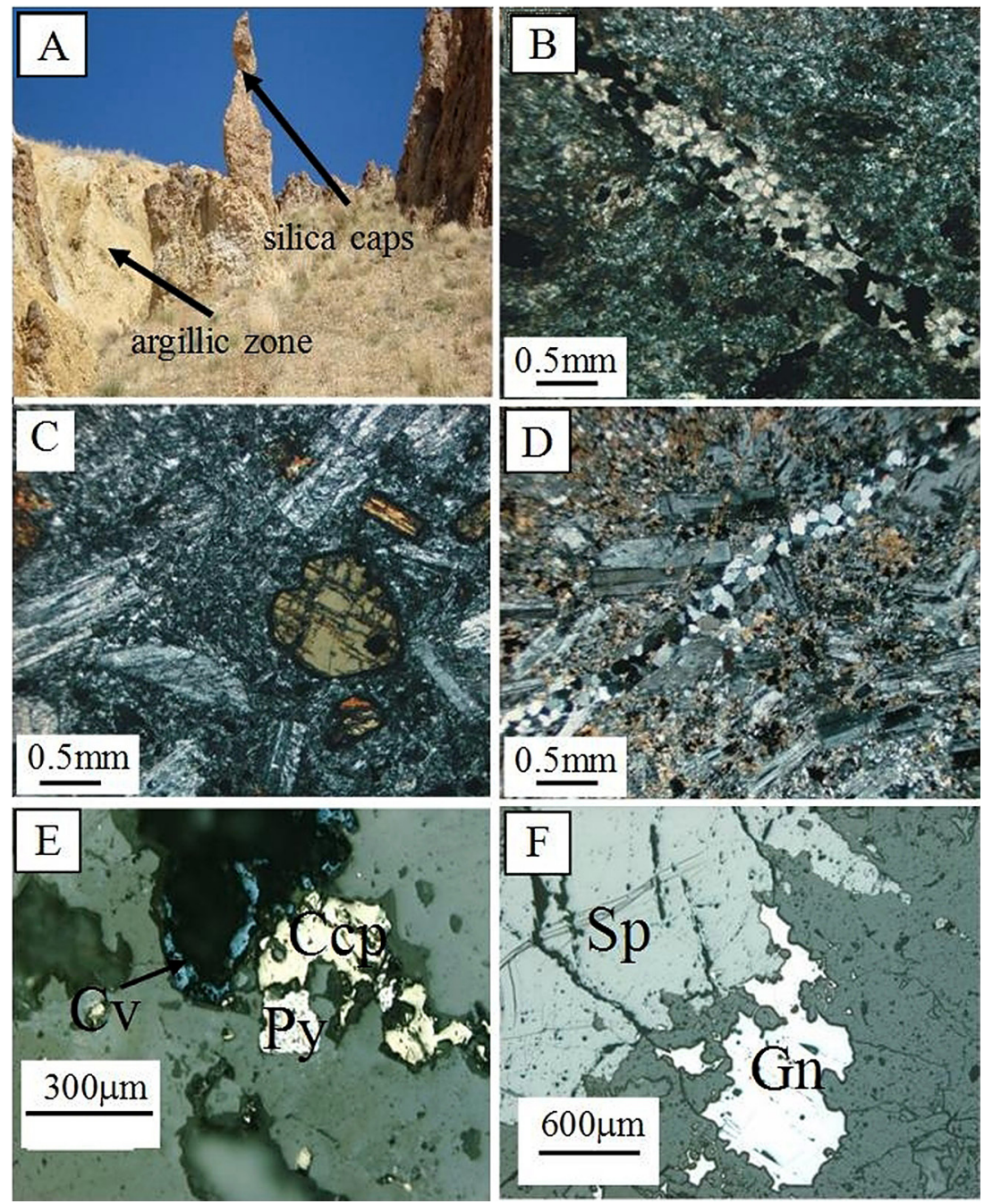

Fig. 4. A - Silica caps in argillic zone; B - Borehole Hen 03-105.6 m: highly to moderately phyllic-argillic alteration microporphyritic quartz diorite; C - Borehole Hen 07 - $161.6 \mathrm{~m}$ : moderately propylitic alteration in propyritic andesite. Plagioclase and hornblende microphenocrysts are major constituents of this rock; D - Borehole Hen 04 - 342 m: moderately potassic (biotitic) alteration. Note the parallel feldspar microphenocrysts and quartz+opaque filled vein; E - Paragenesis of chalcopyrite (Ccp), covellite $(\mathrm{Cv})$ and pyrite (Py); F - Sphalerite (Sp) with galena $(\mathrm{Gn})$ in quartz. crystallisation of a quartz-kaolinite-montmorillonite-chlorite assemblage. In general, it is marked by the complete replacement of plagioclase and K-feldspar. Kaolinite and pyrophyllite are typical mineral phases of the advanced argillic alteration as a result of intense acid attack which culminated in complete leaching of alkali cations with destruction of feldspars and mafic silicate phases (Fig. 4B). XRD analyses indicate that kaolinite is the dominant phyllosilicate, accompanied by illite. Advanced argillic alteration is developed mainly in the northern and eastern parts of Hizehjan and in the northern and northwestern part of Sharafabad; it is the most widespread type in the area.

Phyllic alteration was found in drill cores in the northeastern, southeastern and southwestern parts of Hizehjan where it is more abundant than other alteration types.

Propylitic alteration is developed mainly in the southern, central and northeastern parts of the area and is seen predominantly in andesitic and trachyandesite (Fig. 4C).
Potassic alteration did not occur at rock surface and generated only in well $4(284 \mathrm{~m})$ where it has a thickness of $70 \mathrm{~m}$ (Fig. 4D). This type of alteration may reflect injection of a younger phase into the quartz monzodiorite.

\subsection{Mineralisation}

Mineralisation occurs both as a porphyry type and an epithermal type. The porphyry deposits occur exclusively along Hizehjan faults in the eastern part of the area. Geostatistical and fractal modelling (concentration-number and concentration-area fractal models) in the Hizehjan-Sharaf Abad area show that there is a direct relationship between $\mathrm{Au}$ mineralisations and faults (Rassi \& Afzal, 2015). Mineralisation in epithermal types mainly includes Au-bearing quartz veins within andesite and trachyandesite, which is controlled by fault distribution and is restricted to quartz veins. The textural setting of ore minerals include disseminations, massive accumulations and as 
Table 4. Summary of microthermometric data and calculated parameters for primary fluid inclusions in mineralised quartz from the Mazra'eh Shadi deposit.

\begin{tabular}{|c|c|c|c|c|c|c|c|c|c|c|c|}
\hline Sample & Phases & $\mathrm{T}_{\mathrm{fm}}{ }^{\circ} \mathrm{C}$ & $\begin{array}{l}\text { Tm, } \\
\text { ice }{ }^{\circ} \mathrm{C}\end{array}$ & $\begin{array}{c}\mathrm{Th}(\mathrm{L}+\mathrm{V}) \\
{ }^{\circ} \mathrm{C}\end{array}$ & $\begin{array}{l}\text { Salinity } \\
\text { Wt. \% }\end{array}$ & $\begin{array}{c}\text { Density } \\
\mathrm{g} / \mathrm{cm}^{3}\end{array}$ & $\begin{array}{l}\text { Homoge- } \\
\text { nised to }\end{array}$ & $\begin{array}{l}\text { Size } \\
\mu \mathrm{m}\end{array}$ & Shape & $\begin{array}{c}\mathrm{Au} \\
(\mathrm{ppb})\end{array}$ & $\begin{array}{l}\mathrm{Zn}+\mathrm{Pb} \\
(\mathrm{ppm})\end{array}$ \\
\hline R-19 & $\mathrm{L}+\mathrm{V}$ & & -0.1 & 308.2 & 0.166 & 0.672 & $\mathrm{~L}$ & 61.1 & Elongate & \multirow{21}{*}{59} & \multirow{21}{*}{1156} \\
\hline R-19 & $\mathrm{L}+\mathrm{V}$ & -21 & -1.3 & 178.9 & 2.143 & 0.908 & $\mathrm{~L}$ & 13.9 & Irregular & & \\
\hline R-19 & $\mathrm{V}+\mathrm{L}$ & & -0.8 & 173.4 & 1.322 & 0.908 & V & 7.3 & Irregular & & \\
\hline R-19 & $\mathrm{V}+\mathrm{L}$ & & -0.7 & 287.1 & 1.158 & 0.733 & $\mathrm{~V}$ & 15.0 & Spherical & & \\
\hline R-19 & $\mathrm{L}+\mathrm{V}$ & & -0.5 & 221.1 & 0.827 & 0.844 & $\mathrm{~L}$ & 12.0 & Spherical & & \\
\hline R-19 & $\mathrm{L}+\mathrm{V}$ & -26 & -2.8 & 201.9 & 4.546 & 0.902 & $\mathrm{~L}$ & 5.2 & Spherical & & \\
\hline R-19 & $\mathrm{L}+\mathrm{V}$ & -23 & -2.3 & 209.5 & 3.757 & 0.887 & $\mathrm{~L}$ & 16.0 & Elongate & & \\
\hline R-19 & $\mathrm{L}+\mathrm{V}$ & & -1.1 & 178.2 & 1.816 & 0.906 & $\mathrm{~V}$ & 6.1 & Elongate & & \\
\hline R-19 & $\mathrm{L}+\mathrm{V}$ & & -1.9 & 204.5 & 3.117 & 0.887 & $\mathrm{~L}$ & 7.4 & Spherical & & \\
\hline R-19 & $\mathrm{L}+\mathrm{V}$ & -22 & -1.6 & 251.7 & 2.632 & 0.818 & $\mathrm{~L}$ & 11.4 & Spherical & & \\
\hline R-19 & $\mathrm{L}+\mathrm{V}$ & -22 & -2.1 & 256.6 & 3.438 & 0.819 & $\mathrm{~L}$ & 16.3 & Spherical & & \\
\hline R-19 & $\mathrm{L}+\mathrm{V}$ & & -1.4 & 231.2 & 2.306 & 0.844 & $\mathrm{~L}$ & 11.3 & Spherical & & \\
\hline R-19 & $\mathrm{L}+\mathrm{V}$ & & -2.6 & 267.3 & 4.232 & 0.81 & $\mathrm{~L}$ & 6.9 & Spherical & & \\
\hline R-19 & $\mathrm{L}+\mathrm{V}$ & & -2 & 210.4 & 3.278 & 0.881 & $\mathrm{~L}$ & 9.7 & Elongate & & \\
\hline R-19 & $\mathrm{L}+\mathrm{V}$ & -23 & -1.8 & 190.5 & 2.956 & 0.9 & $\mathrm{~L}$ & 11.1 & Elongate & & \\
\hline R-19 & $\mathrm{V}+\mathrm{L}$ & -22 & -0.9 & 270.0 & 1.487 & 0.771 & V & 12.1 & Spherical & & \\
\hline R-19 & $\mathrm{V}+\mathrm{L}$ & -22 & -0.4 & 298.3 & 0.662 & 0.702 & V & 31.2 & Elongate & & \\
\hline R-19 & $\mathrm{V}+\mathrm{L}$ & & -0.1 & 255.9 & 0.166 & 0.782 & V & 10.6 & Spherical & & \\
\hline R-19 & $\mathrm{V}+\mathrm{L}$ & & -0.3 & 259.9 & 0.497 & 0.779 & V & 17.2 & Spherical & & \\
\hline R-19 & $\mathrm{V}+\mathrm{L}$ & & -0.6 & 324.1 & 0.993 & 0.647 & V & 16.3 & Elongate & & \\
\hline R-19 & $\mathrm{L}+\mathrm{V}$ & -24 & -2.1 & 189.2 & 3.438 & 0.905 & $\mathrm{~L}$ & 30.8 & Spherical & & \\
\hline R-17 & $\mathrm{L}+\mathrm{V}$ & -21 & -0.6 & 216.1 & 0.993 & 0.853 & $\mathrm{~L}$ & 90.1 & Elongate & \multirow{21}{*}{2420} & \multirow{21}{*}{4173} \\
\hline R-17 & $\mathrm{L}+\mathrm{V}$ & -20 & -0.3 & 211.8 & 0.497 & 0.855 & $\mathrm{~L}$ & 92.8 & Elongate & & \\
\hline R-17 & $\mathrm{L}+\mathrm{V}$ & & -1.1 & 200.3 & 1.816 & 0.881 & $\mathrm{~L}$ & 10.9 & Irregular & & \\
\hline R-17 & $\mathrm{L}+\mathrm{V}$ & & -1.6 & 202.5 & 2.632 & 0.886 & $\mathrm{~L}$ & 15.2 & Spherical & & \\
\hline R-17 & $\mathrm{L}+\mathrm{V}$ & & -0.8 & 192.6 & 1.322 & 0.886 & $\mathrm{~L}$ & 9.8 & Spherical & & \\
\hline R-17 & $\mathrm{L}+\mathrm{V}$ & -23 & -2.1 & 238.6 & 3.438 & 0.846 & $\mathrm{~L}$ & 45.0 & Oval & & \\
\hline R-17 & $\mathrm{L}+\mathrm{V}$ & -25 & -1.8 & 239.7 & 2.956 & 0.840 & $\mathrm{~L}$ & 15.5 & Spherical & & \\
\hline R-17 & $\mathrm{L}+\mathrm{V}$ & -21 & -1.5 & 208.8 & 2.469 & 0.877 & $\mathrm{~L}$ & 13.5 & Irregular & & \\
\hline R-17 & $\mathrm{L}+\mathrm{V}$ & & -1.3 & 160.5 & 2.143 & 0.927 & $\mathrm{~L}$ & 21.7 & Elongate & & \\
\hline R-17 & $\mathrm{L}+\mathrm{V}$ & & -1.9 & 209.8 & 3.117 & 0.881 & $\mathrm{~L}$ & 11.7 & Elongate & & \\
\hline R-17 & $\mathrm{L}+\mathrm{V}$ & -24 & -2.1 & 214.3 & 3.438 & 0.878 & $\mathrm{~L}$ & 4.3 & Spherical & & \\
\hline $\mathrm{R}-17$ & $\mathrm{~L}+\mathrm{V}$ & -22 & -1.5 & 255.6 & 2.469 & 0.809 & $\mathrm{~L}$ & 27.9 & Irregular & & \\
\hline $\mathrm{R}-17$ & $\mathrm{~L}+\mathrm{V}$ & & -1.2 & 193.9 & 1.979 & 0.89 & $\mathrm{~L}$ & 8.8 & Irregular & & \\
\hline R-17 & $\mathrm{L}+\mathrm{V}$ & -22 & -1.9 & 206.6 & 3.117 & 0.885 & $\mathrm{~L}$ & 20.2 & Elongate & & \\
\hline R-17 & $\mathrm{L}+\mathrm{V}$ & -23 & -2.3 & 241.4 & 3.757 & 0.845 & $\mathrm{~L}$ & 20.0 & Elongate & & \\
\hline R-17 & $\mathrm{L}+\mathrm{V}$ & -21 & -2.0 & 252.3 & 3.278 & 0.823 & $\mathrm{~L}$ & 12.1 & Spherical & & \\
\hline $\mathrm{R}-17$ & $\mathrm{~L}+\mathrm{V}$ & & -1.6 & 176.2 & 2.632 & 0.913 & $\mathrm{~L}$ & 9.6 & Spherical & & \\
\hline R-17 & $\mathrm{L}+\mathrm{V}$ & & -0.9 & 248.1 & 1.487 & 0.809 & $\mathrm{~L}$ & 13.0 & Spherical & & \\
\hline $\mathrm{R}-17$ & $\mathrm{~L}+\mathrm{V}$ & -22 & -0.5 & 232.7 & 0.827 & 0.828 & $\mathrm{~L}$ & 9.2 & Spherical & & \\
\hline R-17 & $\mathrm{L}+\mathrm{V}$ & & -0.3 & 228.1 & 0.497 & 0.83 & $\mathrm{~L}$ & 11.9 & Spherical & & \\
\hline R-17 & $\mathrm{L}+\mathrm{V}$ & -21 & -0.9 & 269.5 & 1.487 & 0.773 & $\mathrm{~L}$ & 13.9 & Irregular & & \\
\hline H7-D66 & $\mathrm{L}+\mathrm{V}$ & -22 & -1.8 & 209.1 & 2.956 & 0.88 & $\mathrm{~L}$ & 16.5 & Irregular & \multirow{19}{*}{813} & \multirow{19}{*}{389} \\
\hline H7-D66 & $\mathrm{L}+\mathrm{V}$ & -21 & -1.3 & 210.6 & 2.143 & 0.871 & $\mathrm{~L}$ & 20.0 & Elongate & & \\
\hline H7-D66 & $\mathrm{L}+\mathrm{V}$ & & -0.4 & 225.4 & 0.662 & 0.837 & $\mathrm{~L}$ & 13.1 & Spherical & & \\
\hline H7-D66 & $\mathrm{L}+\mathrm{V}$ & & -0.1 & 221.2 & 0.166 & 0.838 & $\mathrm{~L}$ & 10.6 & $\mathrm{NC}$ & & \\
\hline H7-D66 & $\mathrm{L}+\mathrm{V}$ & -22 & -1 & 302.3 & 1.651 & 0.709 & $\mathrm{~L}$ & 10.3 & Oval & & \\
\hline H7-D66 & $\mathrm{L}+\mathrm{V}$ & & -0.9 & 236.8 & 1.487 & 0.829 & $\mathrm{~L}$ & 7.5 & Elongate & & \\
\hline H7-D66 & $\mathrm{L}+\mathrm{V}$ & -19 & -0.5 & 229.7 & 0.827 & 0.832 & $\mathrm{~L}$ & 18.5 & Irregular & & \\
\hline H7-D66 & $\mathrm{L}+\mathrm{V}$ & -21 & -0.7 & 226.6 & 1.158 & 0.84 & $\mathrm{~L}$ & 12.2 & Spherical & & \\
\hline H7-D66 & $\mathrm{L}+\mathrm{V}$ & -24 & -2.1 & 189.9 & 3.438 & 0.905 & $\mathrm{~L}$ & 10 & Elongate & & \\
\hline H7-D66 & $\mathrm{L}+\mathrm{V}$ & & -1.1 & 220.1 & 1.816 & 0.855 & $\mathrm{~L}$ & 8.8 & Elongate & & \\
\hline H7-D66 & $\mathrm{L}+\mathrm{V}$ & & -1.7 & 231.6 & 2.794 & 0.849 & $\mathrm{~L}$ & 10.6 & NC & & \\
\hline H7-D66 & $\mathrm{L}+\mathrm{V}$ & -26 & -3.1 & 243.3 & 5.012 & 0.855 & $\mathrm{~L}$ & 21.8 & Elongate & & \\
\hline H7-D66 & $\mathrm{L}+\mathrm{V}$ & -21 & -1.6 & 212.5 & 2.632 & 0.873 & $\mathrm{~L}$ & 10.7 & Oval & & \\
\hline H7-D66 & $\mathrm{L}+\mathrm{V}$ & -29 & -3.2 & 222.6 & 5.166 & 0.883 & $\mathrm{~L}$ & 12 & Spherical & & \\
\hline H7-D66 & $\mathrm{L}+\mathrm{V}$ & & -2.9 & 215.1 & 4.702 & 0.888 & $\mathrm{~L}$ & 13.5 & Elongate & & \\
\hline H7-D66 & $\mathrm{L}+\mathrm{V}$ & & -1.8 & 220.6 & 2.956 & 0.866 & $\mathrm{~L}$ & 8.2 & Spherical & & \\
\hline H7-D66 & $\mathrm{L}+\mathrm{V}$ & -27 & -2.5 & 265 & 4.074 & 0.812 & $\mathrm{~L}$ & 15.8 & Spherical & & \\
\hline H7-D66 & $\mathrm{L}+\mathrm{V}$ & & -1.1 & 233.1 & 1.816 & 0.837 & $\mathrm{~L}$ & 7.1 & Spherical & & \\
\hline H7-D66 & $\mathrm{L}+\mathrm{V}$ & -24 & -1.2 & 201.9 & 1.979 & 0.881 & $\mathrm{~L}$ & 10.6 & Elongate & & \\
\hline
\end{tabular}




\begin{tabular}{|c|c|c|c|c|c|c|c|c|c|c|c|}
\hline Sample & Phases & $\mathrm{T}_{\mathrm{fm}}{ }^{\circ} \mathrm{C}$ & $\begin{array}{l}\text { Tm, } \\
\text { ice }{ }^{\circ} \mathrm{C}\end{array}$ & $\begin{array}{c}\mathrm{Th}(\mathrm{L}+\mathrm{V}) \\
{ }^{\circ} \mathrm{C}\end{array}$ & $\begin{array}{l}\text { Salinity } \\
\text { Wt. \% }\end{array}$ & $\begin{array}{l}\text { Density } \\
\mathrm{g} / \mathrm{cm}^{3}\end{array}$ & $\begin{array}{l}\text { Homoge- } \\
\text { nised to }\end{array}$ & $\begin{array}{l}\text { Size } \\
\mu \mathrm{m}\end{array}$ & Shape & $\begin{array}{c}\mathrm{Au} \\
(\mathrm{ppb})\end{array}$ & $\begin{array}{l}\mathrm{Zn+Pb} \\
(\mathrm{ppm})\end{array}$ \\
\hline H7-D61 & $\mathrm{L}+\mathrm{V}$ & & -1.4 & 233.9 & 2.306 & 0.842 & $\mathrm{~L}$ & 8.5 & Spherical & \multirow{19}{*}{314} & \multirow{19}{*}{589} \\
\hline H7-D61 & $\mathrm{L}+\mathrm{V}$ & & -0.5 & 252.7 & 0.827 & 0.795 & $\mathrm{~L}$ & 7.1 & Spherical & & \\
\hline H7-D61 & $\mathrm{L}+\mathrm{V}$ & & -1.9 & 224.5 & 3.117 & 0.862 & $\mathrm{~L}$ & 9.7 & Oval & & \\
\hline H7-D61 & $\mathrm{L}+\mathrm{V}$ & -21 & -0.2 & 244.8 & 0.331 & 0.803 & L & 13.3 & Elongate & & \\
\hline H7-D61 & $\mathrm{L}+\mathrm{V}$ & & -0.7 & 210.4 & 1.158 & 0.863 & $\mathrm{~L}$ & 6.2 & Rectangular & & \\
\hline H7-D61 & $\mathrm{L}+\mathrm{V}$ & & -1 & 218.1 & 1.651 & 0.856 & $\mathrm{~L}$ & 5.8 & Spherical & & \\
\hline H7-D61 & $\mathrm{L}+\mathrm{V}$ & -20 & -0.2 & 234.2 & 0.331 & 0.819 & L & 8.6 & Elongate & & \\
\hline H7-D61 & $\mathrm{L}+\mathrm{V}$ & -21 & -0.4 & 196.1 & 0.662 & 0.877 & $\mathrm{~L}$ & 12.7 & Elongate & & \\
\hline H7-D61 & $\mathrm{L}+\mathrm{V}$ & & -0.4 & 213.3 & 0.662 & 0.854 & L & 6.4 & Irregular & & \\
\hline H7-D61 & $\mathrm{L}+\mathrm{V}$ & & -1.7 & 176.7 & 2.794 & 0.915 & $\mathrm{~L}$ & 11.2 & Irregular & & \\
\hline H7-D61 & $\mathrm{L}+\mathrm{V}$ & -24 & 2.2 & 216 & 3.598 & 0.877 & L & 14.5 & Spherical & & \\
\hline H7-D61 & $\mathrm{L}+\mathrm{V}$ & & -1.4 & 221.3 & 2.306 & 0.858 & $\mathrm{~L}$ & 6.6 & Elongate & & \\
\hline H7-D61 & $\mathrm{L}+\mathrm{V}$ & -19 & -1.3 & 206.2 & 2.143 & 0.857 & $\mathrm{~L}$ & 6.1 & Oval & & \\
\hline H7-D61 & $\mathrm{L}+\mathrm{V}$ & & -0.4 & 219.3 & 0.662 & 0.845 & L & 6.6 & Irregular & & \\
\hline H7-D61 & $\mathrm{L}+\mathrm{V}$ & & -0.9 & 202.7 & 1.487 & 0.876 & L & 9.7 & Spherical & & \\
\hline H7-D61 & $\mathrm{L}+\mathrm{V}$ & & -1.5 & 250.9 & 2.469 & 0.817 & L & 6.1 & Spherical & & \\
\hline H7-D61 & $\mathrm{L}+\mathrm{V}$ & -20 & -0.9 & 219.1 & 1.487 & 0.853 & L & 12.1 & Irregular & & \\
\hline H7-D61 & $\mathrm{L}+\mathrm{V}$ & & -2.2 & 230.4 & 3.598 & 0.859 & L & 6.6 & Irregular & & \\
\hline H7-D61 & $\mathrm{L}+\mathrm{V}$ & & -1.3 & 218.5 & 2.143 & 0.861 & L & 6.4 & Irregular & & \\
\hline H7-D155 & $\mathrm{L}+\mathrm{V}$ & -25 & -1.7 & 233.7 & 2.794 & 0.847 & L & 10.8 & Irregular & \multirow{10}{*}{52} & \multirow{10}{*}{1474} \\
\hline H7-D155 & $\mathrm{L}+\mathrm{V}$ & -19 & -0.5 & 219.3 & 0.827 & 0.847 & $\mathrm{~L}$ & 8 & Oval & & \\
\hline H7-D155 & $\mathrm{L}+\mathrm{V}$ & & -1.5 & 225.5 & 2.469 & 0.855 & L & 7.8 & Oval & & \\
\hline H7-D155 & $\mathrm{L}+\mathrm{V}$ & & -0.6 & 259.5 & 0.993 & 0.785 & L & 8.5 & Spherical & & \\
\hline H7-D155 & $\mathrm{L}+\mathrm{V}$ & & -0.5 & 240.2 & 0.827 & 0.815 & L & 7.4 & Spherical & & \\
\hline H7-D155 & $\mathrm{L}+\mathrm{V}$ & -21 & -0.3 & 257.5 & 0.497 & 0.782 & L & 5.5 & Spherical & & \\
\hline H7-D155 & $\mathrm{L}+\mathrm{V}$ & & -0.4 & 280.7 & 0.662 & 0.74 & L & 7.9 & Oval & & \\
\hline H7-D155 & $\mathrm{L}+\mathrm{V}$ & -22 & -0.5 & 241.6 & 0.827 & 0.813 & L & 4.9 & Elongate & & \\
\hline H7-D155 & $\mathrm{L}+\mathrm{V}$ & & -0.9 & 260.3 & 1.487 & 0.789 & L & 8.7 & Spherical & & \\
\hline H7-D155 & $\mathrm{L}+\mathrm{V}$ & -22 & -1.2 & 218.8 & 1.979 & 0.859 & $\mathrm{~L}$ & 8.8 & Elongate & & \\
\hline H8-D65 & $\mathrm{L}+\mathrm{V}$ & & -0.6 & 229.3 & 0.993 & 0.834 & L & 14.2 & Spherical & \multirow{16}{*}{70} & \multirow{16}{*}{368} \\
\hline H8-D65 & $\mathrm{L}+\mathrm{V}$ & & -0.8 & 261.6 & 1.322 & 0.785 & L & 6.6 & Irregular & & \\
\hline H8-D65 & $\mathrm{L}+\mathrm{V}$ & & -1.1 & 249.1 & 1.816 & 0.812 & L & 5.3 & Spherical & & \\
\hline H8-D65 & $\mathrm{L}+\mathrm{V}$ & & -0.5 & 195.5 & 0.827 & 0.879 & L & 29.1 & Spherical & & \\
\hline H8-D65 & $\mathrm{L}+\mathrm{V}$ & & -0.4 & 267.9 & 0.662 & 0.766 & L & 37 & Oval & & \\
\hline H8-D65 & $\mathrm{L}+\mathrm{V}$ & & -0.1 & 208.1 & 0.166 & 0.856 & L & 12.8 & Elongate & & \\
\hline H8-D65 & $\mathrm{L}+\mathrm{V}$ & & -0.4 & 251.6 & 0.662 & 0.795 & L & 13.8 & Spherical & & \\
\hline H8-D65 & $\mathrm{L}+\mathrm{V}$ & & -0.3 & 232.4 & 0.497 & 0.824 & L & 7.9 & Oval & & \\
\hline H8-D65 & $\mathrm{L}+\mathrm{V}$ & & -0.5 & 205.4 & 0.827 & 0.866 & $\mathrm{~L}$ & 10.8 & Elongate & & \\
\hline H8-D65 & $\mathrm{L}+\mathrm{V}$ & & -0.9 & 220.5 & 1.487 & 0.852 & L & 16.4 & Elongate & & \\
\hline H8-D65 & $\mathrm{L}+\mathrm{V}$ & & -1.4 & 252.1 & 2.306 & 0.812 & L & 6.9 & Irregular & & \\
\hline H8-D65 & $\mathrm{L}+\mathrm{V}$ & & -1.7 & 217.7 & 2.794 & 0.868 & L & 8.2 & Elongate & & \\
\hline H8-D65 & $\mathrm{L}+\mathrm{V}$ & & -0.7 & 193.8 & 1.158 & 0.884 & $\mathrm{~L}$ & 8.7 & Elongate & & \\
\hline H8-D65 & $\mathrm{L}+\mathrm{V}$ & & -0.2 & 199.6 & 0.331 & 0.87 & L & 11.2 & Elongate & & \\
\hline H8-D65 & $\mathrm{L}+\mathrm{V}$ & & -1.5 & 239.4 & 2.469 & 0.834 & L & 24.8 & Spherical & & \\
\hline H8-D65 & $\mathrm{L}+\mathrm{V}$ & & -1.2 & 227.1 & 1.979 & 0.847 & $\mathrm{~L}$ & 21.3 & Spherical & & \\
\hline Average & & -22 & -1.1 & 227.9 & 1.94 & 0.84 & & 14.4 & & & \\
\hline Max & & -19 & 2.2 & 324.1 & 5.17 & 0.93 & & 92.8 & & & \\
\hline Min & & -29 & -3.2 & 160.5 & 0.17 & 0.65 & & 4.3 & & & \\
\hline
\end{tabular}

a matrix of hydrothermal breccia. The main minerals of the epithermal type are galena, sphalerite, pyrite and chalcopyrite (Fig. 4E-F). Geochemical analyses of altered andesite and trachyandesite in northern Mazra'eh Shadi have demonstrated distinct anomalies of $\mathrm{Au}, \mathrm{Pb}, \mathrm{Ag}$, $\mathrm{Cu}$ and $\mathrm{Zn}$. Ore and gangue mineral as- semblages at Mazra' eh Shadi are typical of epithermal deposits. The superficial ore minerals are oxidised Fe-oxide/hydroxide and pyrite, whereas galena, pyrite and chalcopyrite are found in deeper horizons of the mine. The chemical analyses show maximum values of $\mathrm{Zn}$ (1650 ppm), Ag (1.4 ppm), Cu (745 ppm) 

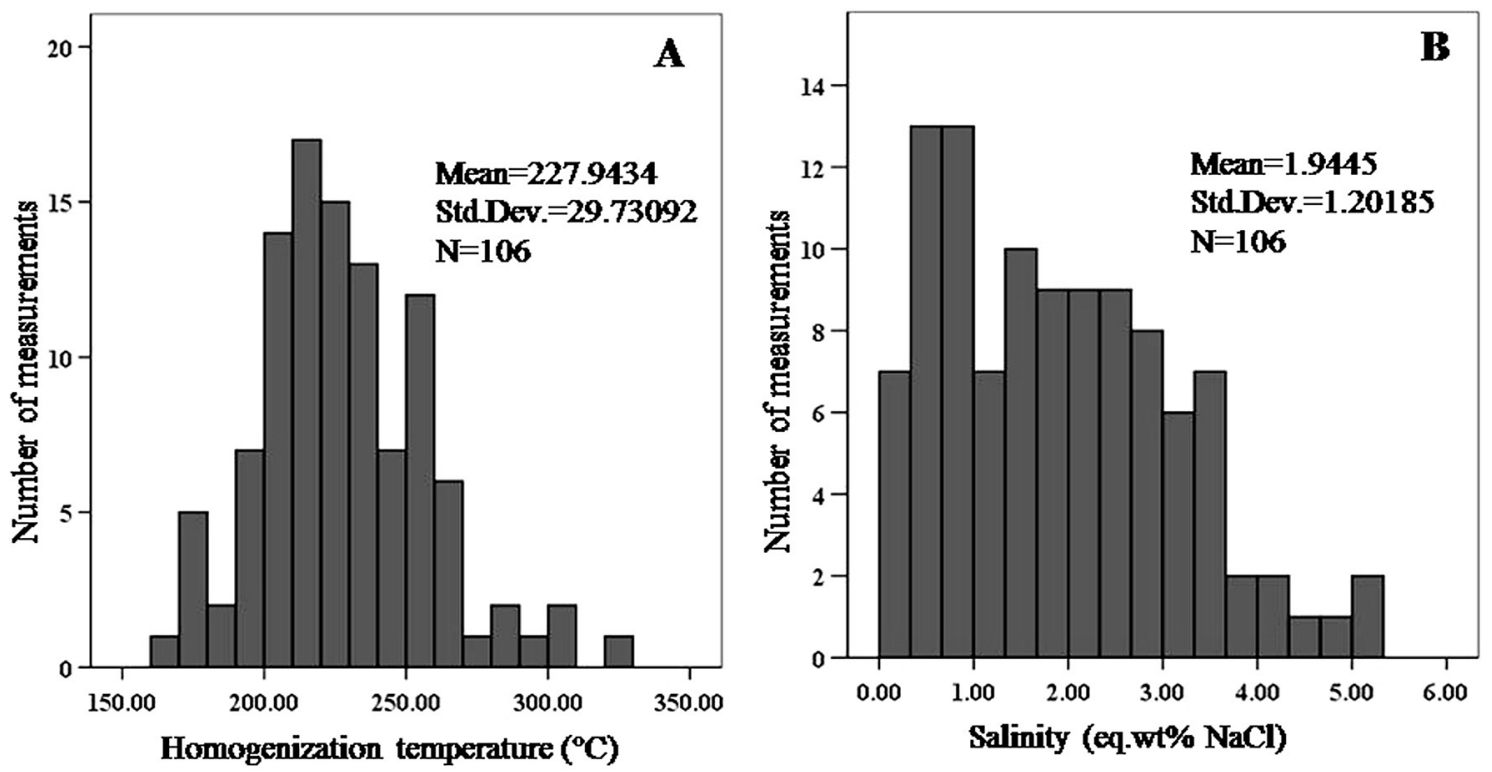

Fig. 5. Histogram of homogenisation temperature and salinity for 106 studied liquid-rich (LV) and vapour-rich (VL) fluid inclusions.

and Mo $(87 \mathrm{ppm})$ in the quartz monzodiorite (Table 1). The maximum concentration of $\mathrm{Au}$ is $17100 \mathrm{ppb}$, of $\mathrm{Pb}$ it is 21100 ppm, Ag 9.43 ppm, Cu 611 ppm and $\mathrm{Zn}$ $333 \mathrm{ppm}$ in the veins within the silicified zones (Table 2). Pyrite is the most abundant sulphide mineral. Alteration associated with mineralisation is confined to thin halos of silicified rocks adjacent to the veins, bordered by argillic and propylitic zones outwards.

\subsection{Fluid inclusions}

A total of 106 microthermometric measurements were performed on primary inclusions. Homogenisation temperatures of inclusions in quartz from drill core samples and surface outcrops vary be- tween $160-324$ and $176-302^{\circ} \mathrm{C}$, respectively, with an average of $228^{\circ} \mathrm{C}$, which were commonly observed either as inclusions along growth zones or isolated single inclusions in crystals. The ice-melting temperatures from quartz vary from 2.2 to $-3.2^{\circ} \mathrm{C}$, which correspond to a salinity range from 0.17 to 5.17 wt. \% NaCI equivalent, with an average of 1.9 wt. \% NaCI equivalent. The inclusions have a wide size range from 5 to $90 \mu \mathrm{m}$, with an average of 14 $\mu \mathrm{m}$. The largest fluid inclusion in the study area has been observed in outcrop sample R17, which measures $93 \mu \mathrm{m}$. Microthermometric data and calculated parameters such as salinities, homogenisation temperatures, ice-melting temperatures, density and size for fluid inclusions are summarised in Table 4 and graphically illustrated in Figure 5. In
Fig. 6. Homogenisation temperature vs. frequency diagram in coexistence of vapour-rich and liquid-rich inclusions reflecting boiling temperature of fluid inclusion in the Mazra'eh Shadi deposit.

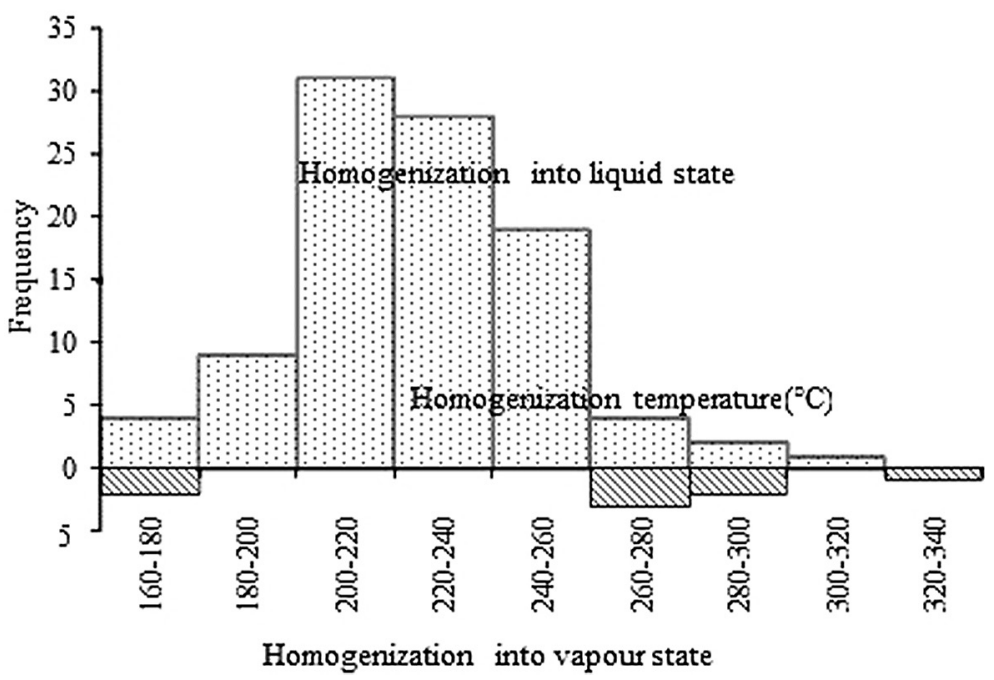


Mazra'eh Shadi, quartz veins containing co-existing liquid-rich and vapour-rich inclusions, as a strong evidence of boiling during hydrothermal evolution, have relatively higher gold grades (Fig. 6).

\section{Discussion}

In order to determine element distribution in the area, image maps were prepared for $\mathrm{Au}, \mathrm{Cu}$ and other elements (Fig. 7). The surface map indicates that $\mathrm{Au}$ forms a strong anomaly only in one area in the southwestern region, in veins within the silicified zones. Gold mineralisation is mostly associated with $\mathrm{Ag}$, as observed as veins within silicified zones in Mazra'eh Shadi. The presence of two different anomalies in $\mathrm{Cu}$ distribution (Fig. 7) may indicate two different phases of $\mathrm{Cu}$ mineralisation in the study area. Pearson correlation coefficients greater than 0.5 are significant (dependent upon the number of samples; in the present paper 0.5 for 16-23 sample), whereas Pearson correlation coefficients greater than 0.8 indicate a strong correlation between the two variables. Pearson correlation coefficients calculations and factor analysis of geochemical data were performed using the statistical package for social science (SPSS) (Nie et al., 1975). Factor analysis, which is important in the evaluation of geological studies, is a series of processes for investigating the dependence structure of multivariate models with fewer unobserved variables (Davis, 1986). The reasons that maintain the dependence among the variables are considered factors and thus their effects on the variables could be examined separately (Howarth, 1993; Tuysuz \& Yaylali, 2005). Factor analysis is a statistical method that reduces numerous variables to a lesser number of mutually uncorrelated factors that can be related to similar origins or sources. Factor analysis also identifies the minimum number of variables necessary to account for the variation (\% variance) in a data set (McLemore, 2008). Pearson correlation coefficients and factor analysis for quartz monzodiorite and Au-bearing quartz veins are summarised in Tables 5-8.

Statistical analyses confirm a significant correlation between $\mathrm{Au}, \mathrm{Ag}, \mathrm{Pb}, \mathrm{Zn}$ and a strong correlation between $\mathrm{Pb}-\mathrm{Ag}$ and $\mathrm{Pb}-\mathrm{Zn}$ in the $\mathrm{Au}$-bearing quartz veins (Table 6). Evidence from geology, alteration, mineralisation, geochemical investigations and grades of elements indicate that $\mathrm{Au}$ and $\mathrm{Ag}$ contents are high in quartz veins within the silicified zones in Mazra' eh Shadi (Fig. 7; Tables 3, 6-10).

Factor group 1 indicates a strong correlation between $\mathrm{Pb}, \mathrm{Ag}, \mathrm{Sb}$ and As. Factor group 2 indicates a correlation between $\mathrm{Au}, \mathrm{Cu}$ and $\mathrm{Zn}$, while factor group 3 consists of Mo, Sn, S and W. The placement of $\mathrm{Au}$ and $\mathrm{Cu}$ in factor group 2 can be related to similar origins or the fact that these elements were derived from the same source in a subvolcanic unit. Note that $60.07 \%$ of the variation in the data is attributed to factors 1, 2 and 3. In the Au-bearing quartz veins factor group 1 indicates a strong correlation between $\mathrm{Au}, \mathrm{Pb}, \mathrm{Ag}$, $\mathrm{Zn}$ and $\mathrm{W}$. Factor group 2 indicates a correlation between $\mathrm{Cu}, \mathrm{Te}, \mathrm{Sb}$ and $\mathrm{Zn}$, while factor group 3 consists of Mo and As. Over $69.66 \%$ of the variation in the data is attributed to factors 1, 2 and 3. The separate associations of Au$\mathrm{Ag}-\mathrm{Pb}-\mathrm{Zn}$ and $\mathrm{Cu}$ represented by factor groups 1 and 2 are confirmed by field and production data. Median and arithmetic mean for the samples are considerably different from each other and this indicates a log-normal distribution. If the distribution elements are abnormal, Spearman correlation coefficients have become more important than Pearson correlation coefficients. Based on Spearman correlation coefficients, $\mathrm{Sb}$ and Te can be very good indicators for $\mathrm{Au}, \mathrm{Ag}$ and $\mathrm{Pb}$ epithermal mineralisations (Tables 9-10). The dendrogram shows that $\mathrm{Au}$ with $\mathrm{Cu}$ and $\mathrm{Zn}$ form the same group in quartz monzodiorite-diorite (Fig. 8). Correlation between $\mathrm{Au}$ and $\mathrm{Cu}$ indicates that these elements have similar concentrations and migrate together using the same pathway (fault) in subvolcanic units. In the Au-bearing quartz veins, Au with $\mathrm{Zn}, \mathrm{Fe}, \mathrm{S}, \mathrm{Ag}$ and $\mathrm{Pb}$ forms one group (Fig. 9).

The image map for $\mathrm{Cu}, \mathrm{Mo}, \mathrm{Pb}, \mathrm{Zn}, \mathrm{Ag}, \mathrm{Ni}, \mathrm{Cd}$ and $\mathrm{Ag}^{*} \mathrm{~Pb}^{*} \mathrm{Zn} / \mathrm{Cu}^{*} \mathrm{Bi}^{*} \mathrm{Co}$ in subvolcanic rocks shows an irregular distribution pattern of elements (Fig. 10). Below the ore halo consists of $\mathrm{Cu}-\mathrm{Bi}-\mathrm{Co}$, while $\mathrm{Ag}-\mathrm{Pb}-\mathrm{Zn}$ forms the upper halo. In normal conditions, from the surface into the deep this ratio must decrease. Plotting of $\mathrm{Ag}^{*} \mathrm{~Pb}^{*} \mathrm{Zn} / \mathrm{Cu}^{*} \mathrm{Bi}^{*} \mathrm{Co}$ of the samples against depth in borehole 3 (Fig. 11) in subvolcanic rocks elucidates two important points: the distribution pattern of this ratio is irregular, has a zigzag shape and shows higher values in the lower part. The values of $\mathrm{Ag}^{*} \mathrm{~Pb}^{*} \mathrm{Zn} / \mathrm{Cu}^{*} \mathrm{Bi}^{*} \mathrm{Co}$ have a range of $0.003-2.34$. This diagram shows that these values are regular from the surface to a depth of $246 \mathrm{~m}$ and then become considerably irregular in the deeper part. It is possible that another pluton intruded within the first pluton in the region and cause the irregular shape and $\mathrm{Ag}^{*} \mathrm{~Pb} \mathrm{Zn}^{*} \mathrm{Cu}^{*} \mathrm{Bi}^{*}$ Co ratio in relation to depth.

In order to illustrate element distribution in the area, we have prepared longitudinal sections for the veins using chemical assays and their corresponding " $x$ " (Universal Transverse Mercator (UTM) easting) and " $y$ " (elevation) co-ordinates (Fig. 12). 

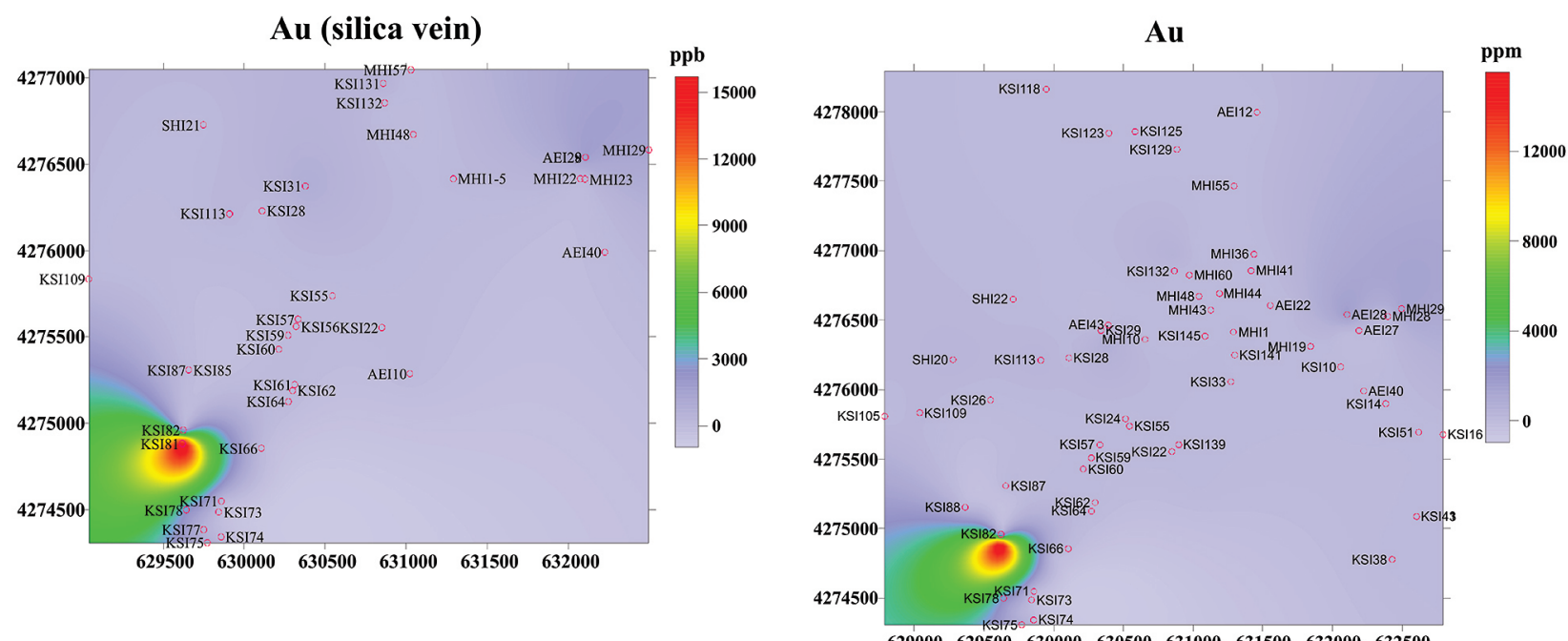

\section{$\mathbf{P b}$}

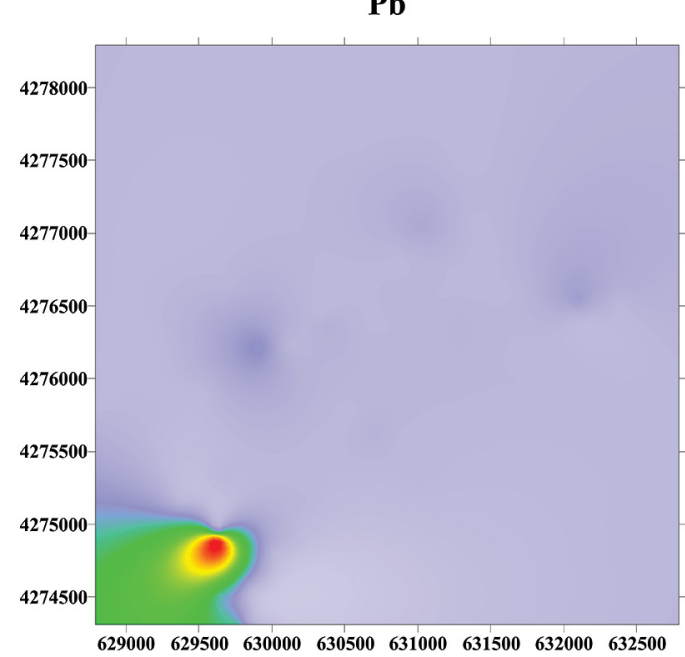

Mo

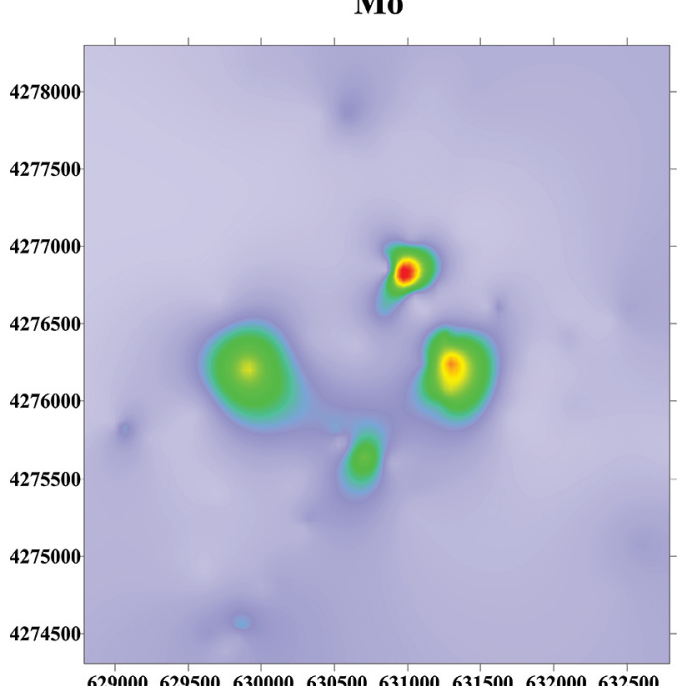

ppm

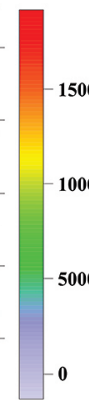

ppm
Ag

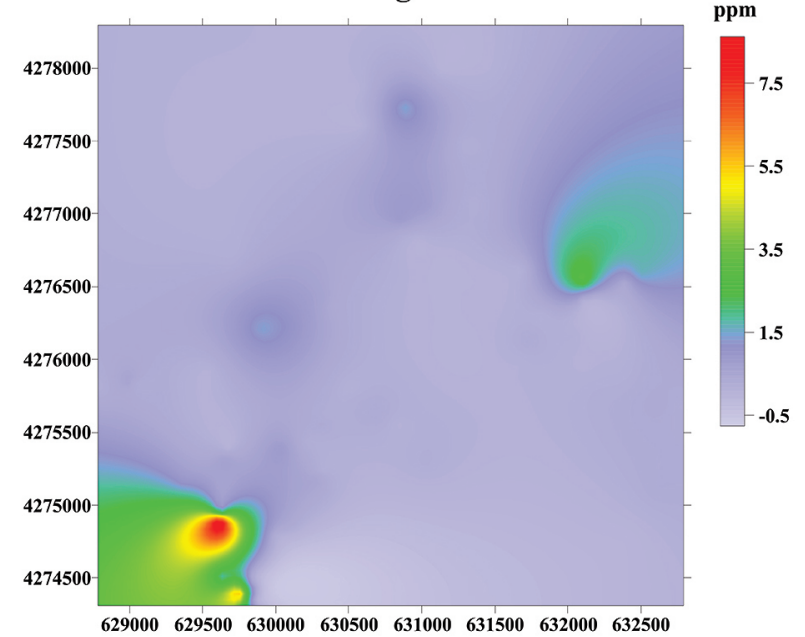

$\mathrm{Cu}$

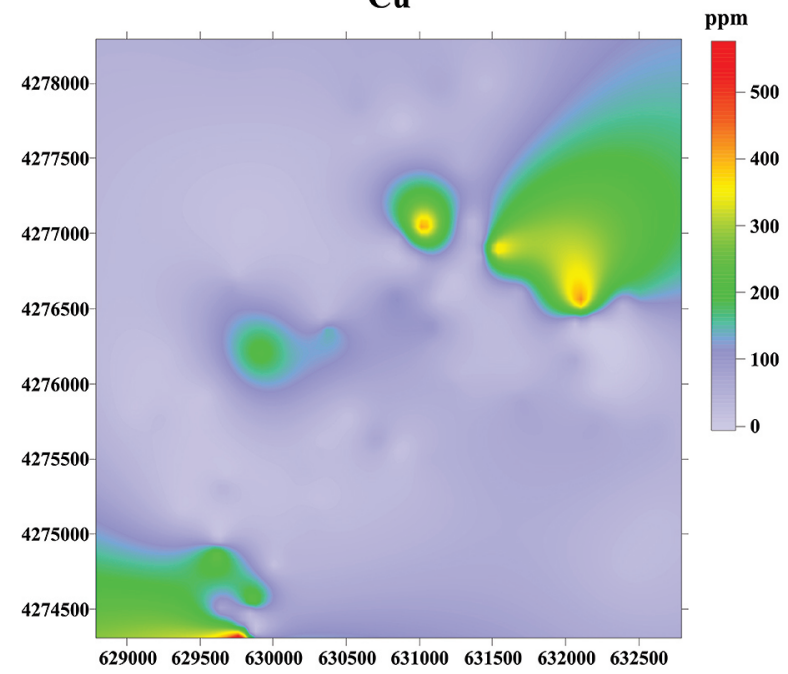

Fig. 7. Image map for $\mathrm{Au}, \mathrm{Pb}, \mathrm{Ag}, \mathrm{Mo}$ and $\mathrm{Cu}$. Axis " $\mathrm{x}$ ” UTM easting and Axis " $\mathrm{y}$ " UTM northing. Location of sampled sites for $\mathrm{Pb}, \mathrm{Ag}, \mathrm{Mo}, \mathrm{Cu}$ maps is the same as on upper right map. 

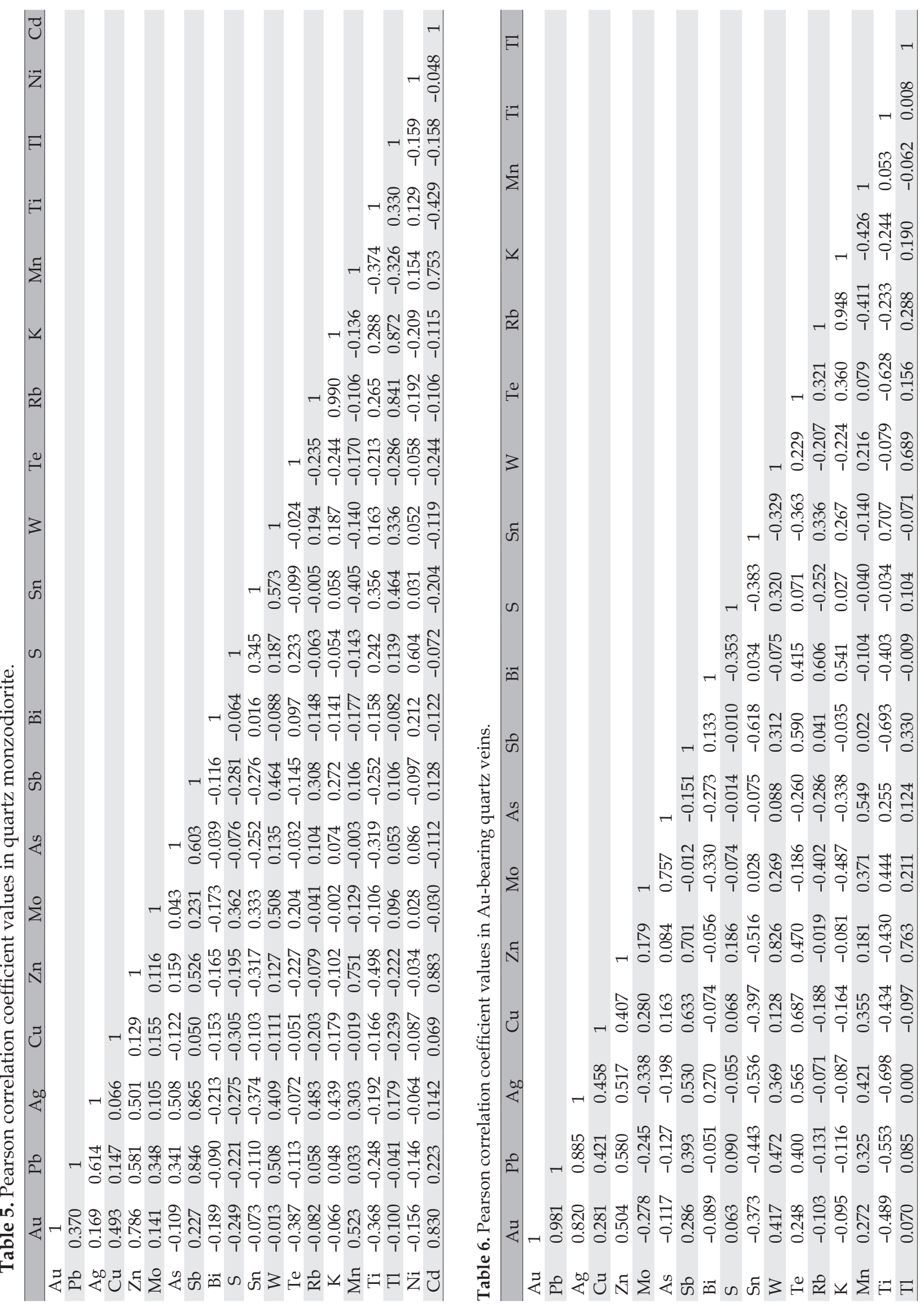
Table 7. Varimax rotated factor analyses (R mode) of assays of quartz monzodiorite.

\begin{tabular}{|ccccc|}
\hline & Factor 1 & Factor 2 & Factor 3 & Factor 4 \\
\hline $\mathrm{Au}$ & 0.073 & 0.923 & -0.09 & 0.116 \\
$\mathrm{Cu}$ & -0.105 & 0.675 & -0.044 & -0.207 \\
$\mathrm{Mo}$ & 0.152 & 0.246 & 0.824 & -0.13 \\
$\mathrm{~Pb}$ & 0.759 & 0.401 & 0.214 & 0.001 \\
$\mathrm{Ag}$ & 0.894 & 0.131 & -0.035 & 0.066 \\
\hline $\mathrm{Te}$ & -0.039 & -0.336 & 0.334 & -0.738 \\
\hline $\mathrm{Zn}$ & 0.481 & 0.686 & -0.095 & -0.057 \\
$\mathrm{Sb}$ & 0.954 & 0.188 & 0.049 & 0.07 \\
$\mathrm{~S}$ & -0.247 & -0.282 & 0.628 & -0.075 \\
\hline $\mathrm{As}$ & 0.727 & -0.252 & -0.089 & -0.035 \\
\hline $\mathrm{Bi}$ & -0.091 & -0.309 & -0.164 & -0.075 \\
\hline $\mathrm{W}$ & 0.427 & 0.027 & 0.71 & 0.349 \\
\hline $\mathrm{Tl}$ & 0.088 & -0.24 & 0.225 & 0.785 \\
\hline $\mathrm{Sn}$ & -0.355 & -0.061 & 0.652 & 0.54 \\
\hline
\end{tabular}

Table 8. Varimax rotated factor analyses (R-mode) of assays of Au-bearing quartz veins.

\begin{tabular}{|ccccc|}
\hline & Factor 1 & Factor 2 & Factor 3 & Factor 4 \\
\hline $\mathrm{Au}$ & 0.969 & 0.085 & -0.109 & 0.052 \\
\hline $\mathrm{Cu}$ & 0.179 & 0.775 & 0.255 & -0.043 \\
\hline $\mathrm{Mo}$ & -0.186 & 0.103 & 0.942 & 0.02 \\
$\mathrm{~Pb}$ & 0.944 & 0.247 & -0.103 & 0.062 \\
$\mathrm{Te}$ & 0.146 & 0.846 & -0.267 & -0.092 \\
$\mathrm{Ag}$ & 0.798 & 0.458 & -0.244 & -0.13 \\
\hline $\mathrm{Sb}$ & 0.223 & 0.848 & -0.029 & 0.056 \\
\hline $\mathrm{S}$ & -0.039 & 0.07 & -0.117 & 0.918 \\
\hline $\mathrm{As}$ & -0.039 & -0.044 & 0.875 & -0.009 \\
\hline $\mathrm{Bi}$ & -0.101 & 0.322 & -0.44 & -0.552 \\
\hline $\mathrm{Sn}$ & -0.302 & -0.579 & 0.019 & -0.437 \\
\hline $\mathrm{Zn}$ & 0.51 & 0.269 & 0.265 & 0.427 \\
\hline \% & 0.533 & 0.584 & 0.233 & 0.306 \\
\hline
\end{tabular}
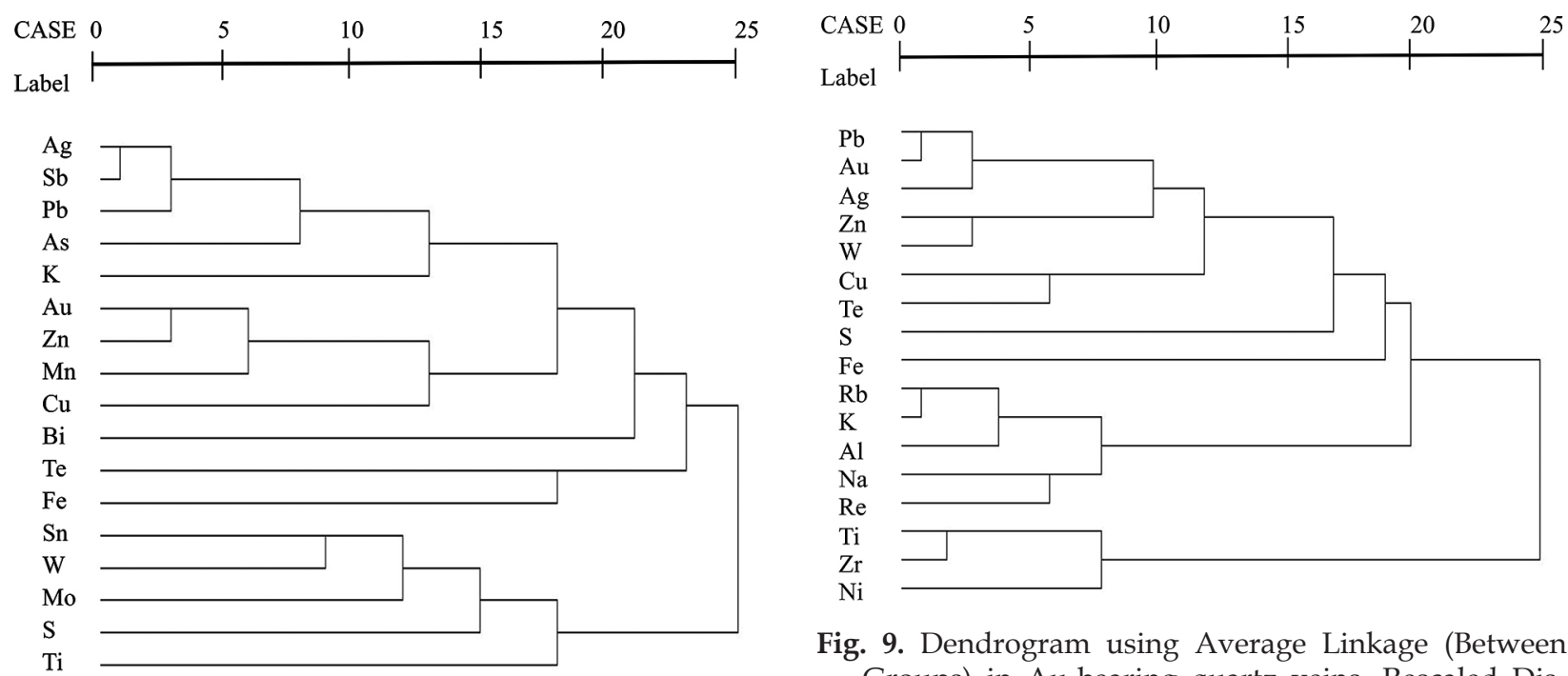

Fig. 8. Dendrogram using Average Linkage (Between Groups) in subvolcanic rocks. Rescaled Distance Cluster Combine.

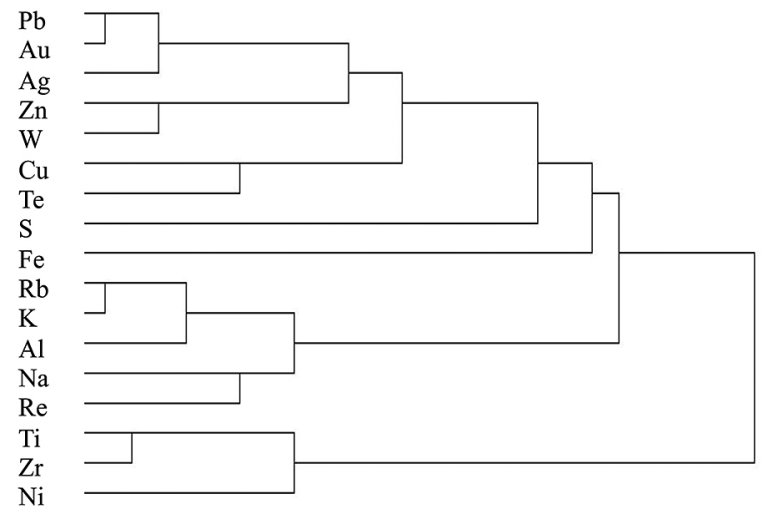

Fig. 9. Dendrogram using Average Linkage (Between Groups) in Au-bearing quartz veins. Rescaled Distance Cluster Combine. 

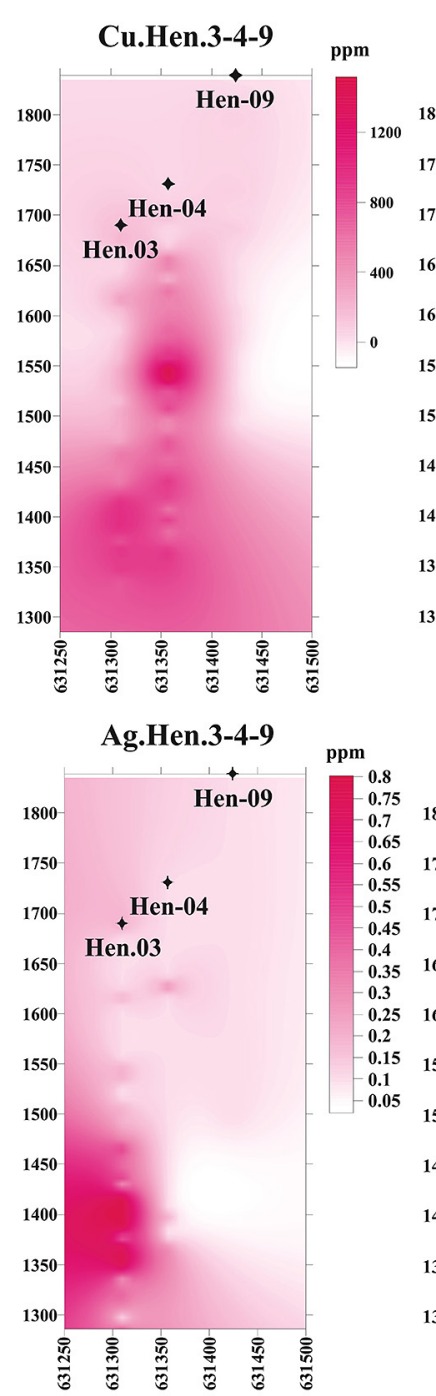
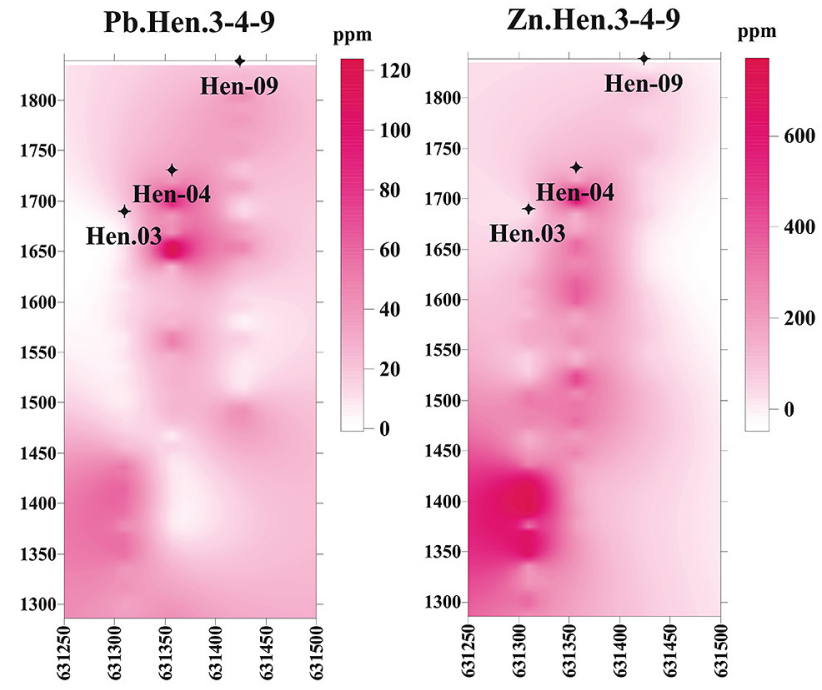

Cd.Hen.3-4-9

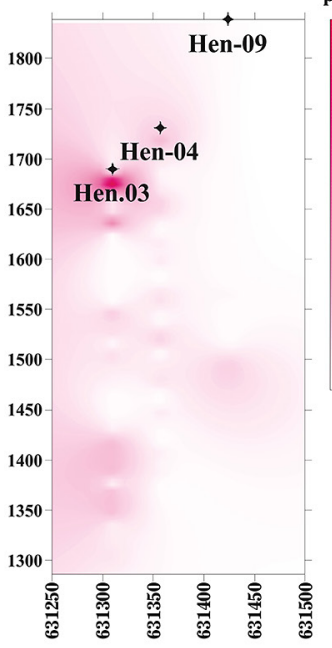

Fig. 10. Image map for $\mathrm{Cu}, \mathrm{Mo}, \mathrm{Pb}, \mathrm{Zn}, \mathrm{Ag}, \mathrm{Ni}, \mathrm{Cd}$ and $\mathrm{Ag} * \mathrm{~Pb} * \mathrm{Zn} / \mathrm{Cu}^{*} \mathrm{Bi}^{*} \mathrm{Co}$ in subvolcanic rocks. Elevation in metres above sea level (m a.s.l.) and distance is in metres.

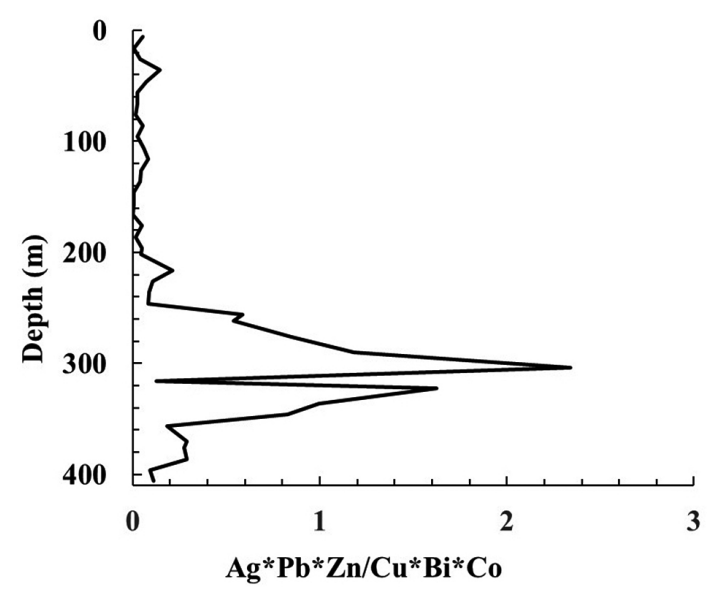

Fig. 11. Plotting of $\mathrm{Ag}^{*} \mathrm{~Pb}{ }^{*} \mathrm{Zn} / \mathrm{Cu}^{*} \mathrm{Bi}^{*} \mathrm{Co}$ of samples against depth in borehole 3 in subvolcanic rocks.
Variations in metal concentrations and metal ratios occur in Mazra' eh Shadi with depth. These variations are evidence of geochemical zoning. Longitudinal sections demonstrate that $\mathrm{Cu}, \mathrm{Pb}, \mathrm{Zn}$ and $\mathrm{Mo}$ are concentrated at depth, whereas $\mathrm{Au}$ and $\mathrm{Ag}$ occur at shallower levels, above the base metal zones. This observation contradicts the typical zoning pattern caused by boiling in epithermal veins (Buchanan, 1981; Grancea et al., 2002). In the Purisima-Colon vein system (Pachuca, Mexico), base metals are concentrated at depth, and there is an intermediate-depth zone with high Ag grades overlapping the base metal zone (Dreier, 2005). The presence of adularia, bladed calcite, and coexisting liquid-rich and vapour-rich inclusions are evidence of local boiling (Browne \& Ellis, 1970; Browne, 1978; Hedenquist et al., 2000; Simmons \& Browne, 2000). Plotting of Pb, $\mathrm{Zn}$ and $\mathrm{Au}$ against depth in Au-bearing quartz veins 

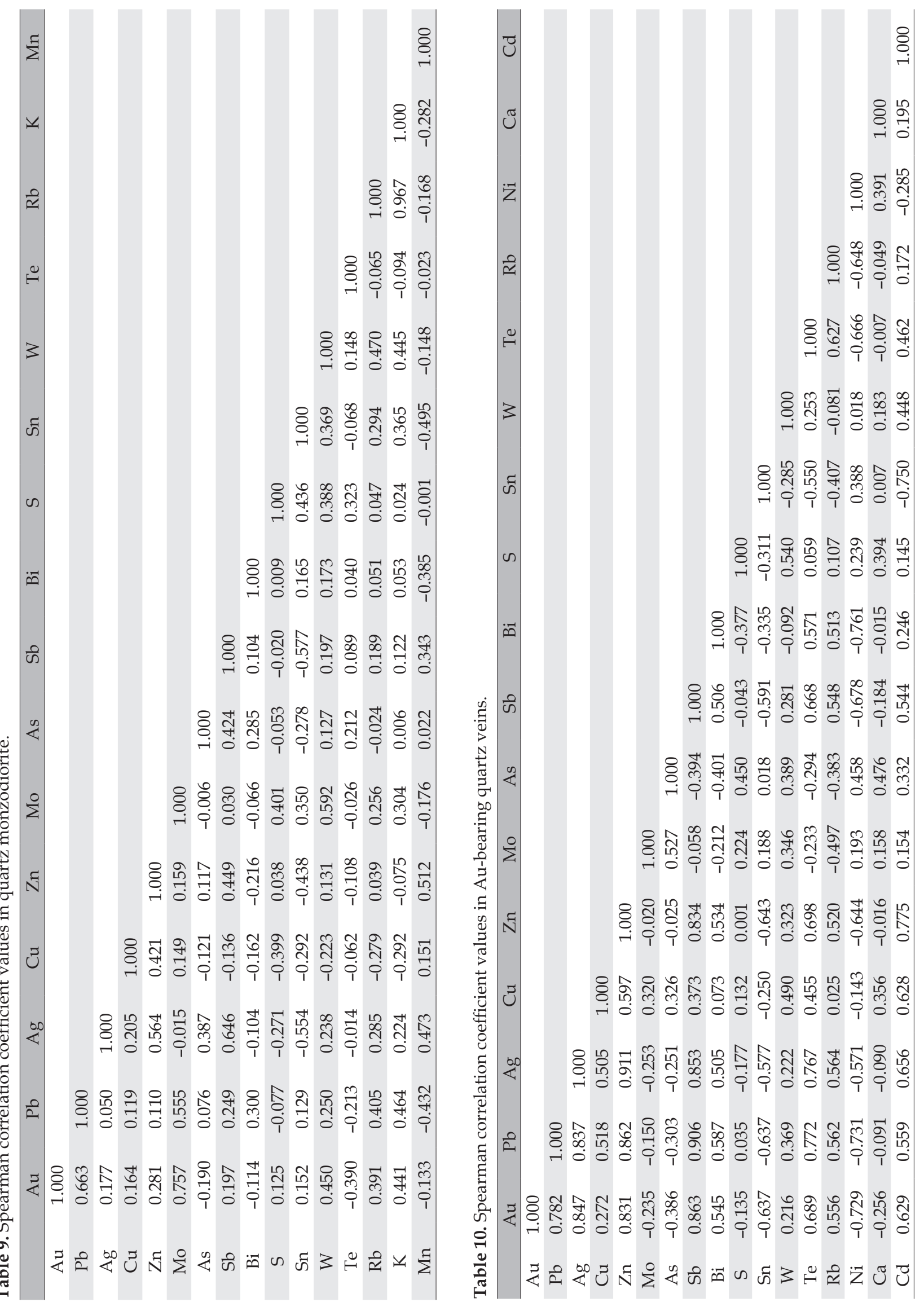
Cu.Hen.6-7-8-11

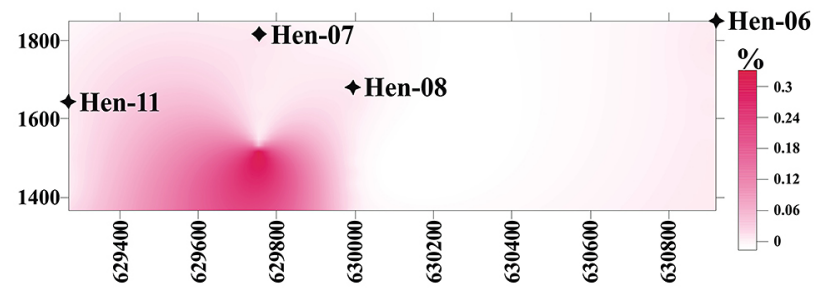

Pb-Hen.6-7-8-11
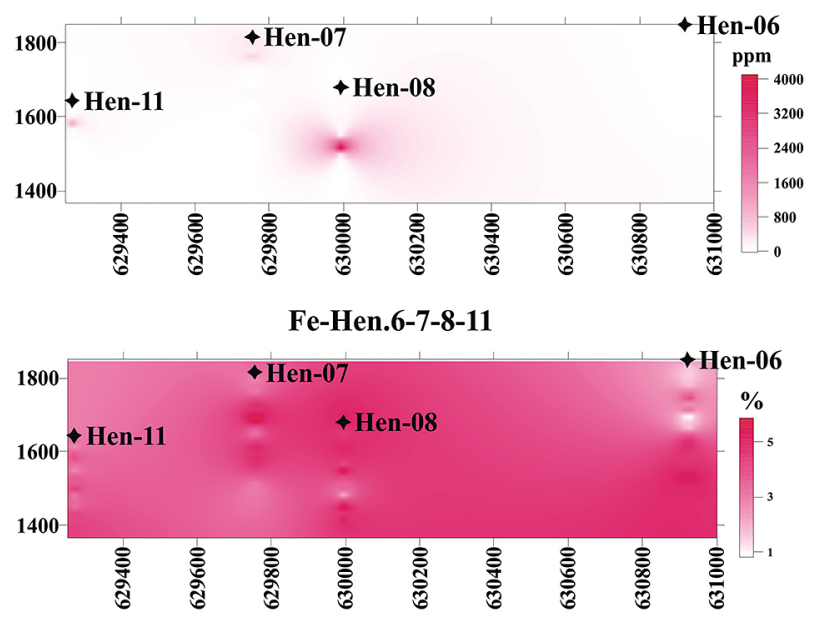

Cu+As.Hen.6-7-8-11

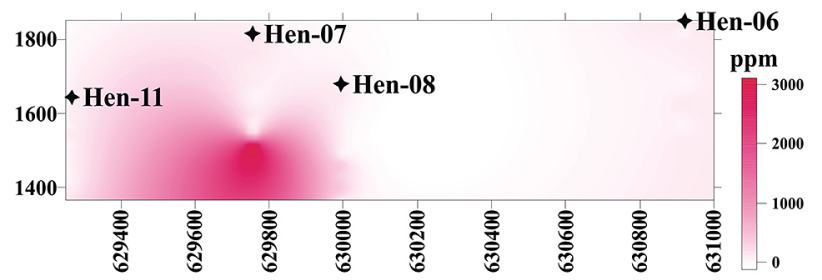

Au.Hen.6-7-8-11

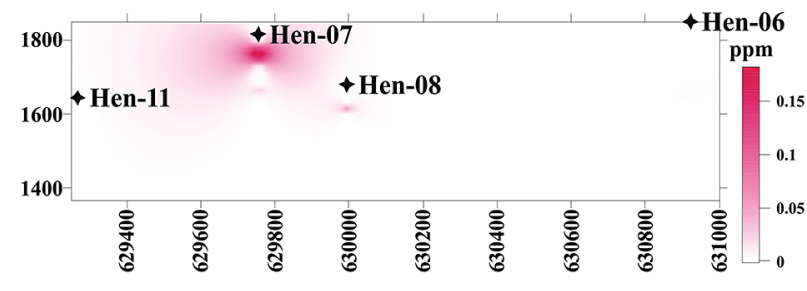

Mo.Hen.6-7-8-11

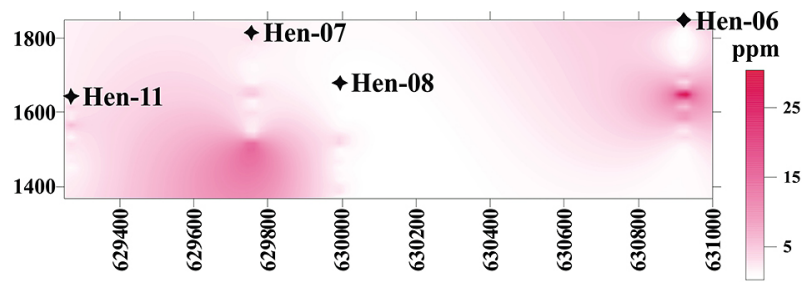

Ag-Hen.6-7-8-11

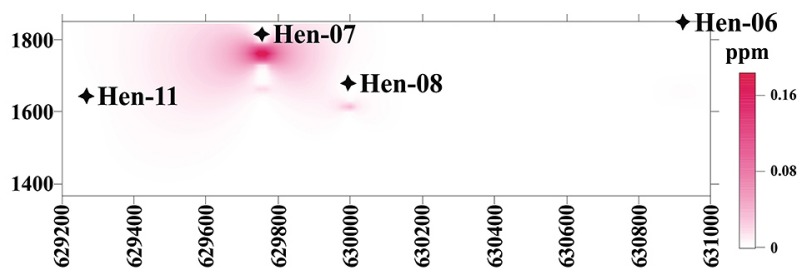

$\mathrm{Pb}+\mathrm{Zn} . H e n .6-7-8-11$

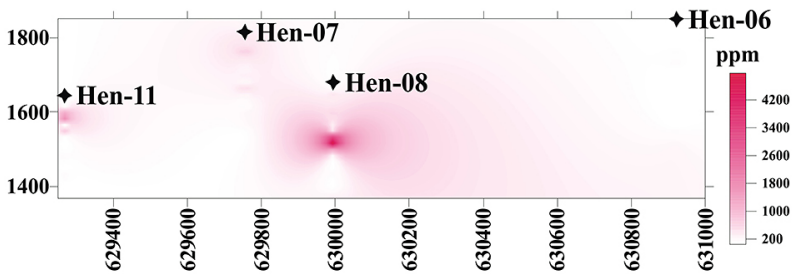

Fig. 12. Image map for $\mathrm{Cu}, \mathrm{Au}, \mathrm{Pb}, \mathrm{Mo}, \mathrm{Fe}, \mathrm{Ag}, \mathrm{Cu}+\mathrm{As}$ and $\mathrm{Pb}+\mathrm{Zn}$ in Au-bearing quartz veins. Axis " $\mathrm{x}$ ” UTM easting and axis " $y$ " elevation in metres above sea level (m a.s.l.).

shows that these elements have similar behaviours. Gold and silver concentrations increased with depth to the 45- and 155-m-levels (Fig. 13). The highest Au and Ag concentration in the study area was obtained from the phyllic-silicic alteration (Fig. 14).

In most epithermal deposits, pyrite or an iron oxide phase and adularia are common gangue minerals (Sillitoe \& Hedenquist, 2003). Pyrite precipitation also promotes $\mathrm{Au}$ deposition by removing the complexing agent $(S)$ responsible for $\mathrm{Au}$ transportation (Henley \& Brown, 1985) according to equation (1)

$$
\mathrm{Fe}^{2+}+2 \mathrm{Au}(\mathrm{HS})^{2-}=2 \mathrm{Au}(\mathrm{s})+\mathrm{FeS}_{2}(\mathrm{~s})+2 \mathrm{H}^{+}+2 \mathrm{HS}^{-}
$$

Most epithermal ore-forming fluids probably are not saturated in Au initially, but rather achieve saturation and deposit $\mathrm{Au}$ as a result of changes in chemical and physical conditions at the site of deposition. In summary, precipitation of Au takes place in response to changes in temperature, pressure, $\mathrm{pH}, \mathrm{Eh}$ and the activity of reduced $\mathrm{S}$. The mechanisms by which Ag is dissolved and transported in epithermal systems, on the other hand, are not as well understood. If $\mathrm{Ag}$ is transported as a sulphide complex, boiling should also cause $\mathrm{Ag}$ to precipitate. However, if $\mathrm{Ag}$ is transported as a chloride complex boiling is less likely to result in Ag precipitation. Recent experimental studies by Pokrovski et al. (2008) at $350-500^{\circ} \mathrm{C}$ (temperatures somewhat higher than those in epithermal environments) have shown that the solubility of Ag in chloride solutions decreases with increasing $\mathrm{pH}$, reflecting a change from transport as a chloride complex in acidic solutions to transport as S-complexes in neutral to basic solutions. Thus, the changes in solution properties described above as 


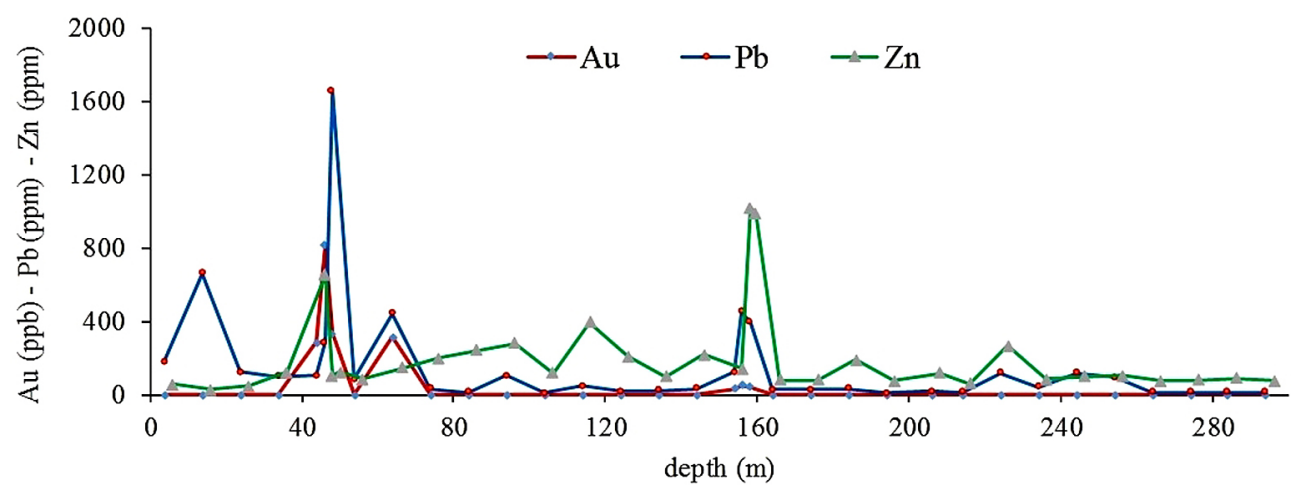

Fig. 13. Distribution pattern of $\mathrm{Au}, \mathrm{Pb}$ and $\mathrm{Zn}$ of ore samples against depth in borehole 7 in $\mathrm{Au}$-bearing quartz veins.
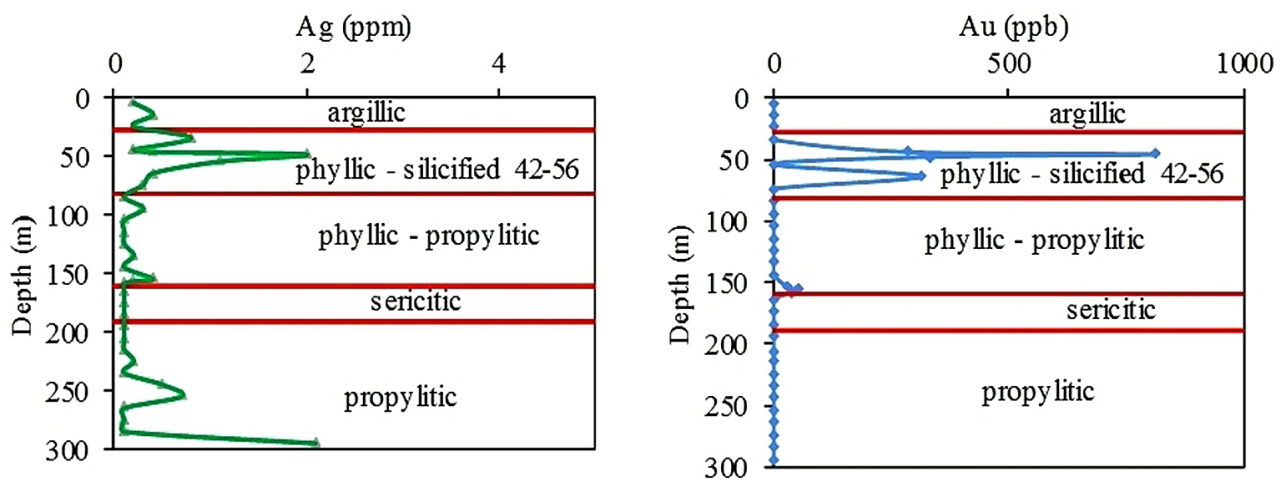

Fig. 14. Plotting of Ag and Au against depth in borehole 7 in Au-bearing quartz veins.

a result of boiling, specifically a change in $\mathrm{pH}$, will also promote Ag deposition if Ag is transported as a sulphur complex. The correlation between $\mathrm{Au}-$ $\mathrm{Ag}$ and the close association of this element in a dendrogram with $\mathrm{Fe}$ and $\mathrm{S}$ in Au-bearing quartz veins (Fig. 9) are due to deposition of this element as a result of pyrite precipitation under temperatures lower than $350^{\circ} \mathrm{C}$.

Silicification is also pervasive in the shallower environment where it develops as a result of cooling and decreasing acidity of hydrothermal fluids as a result water-rock interaction and mixing with surface waters (Sillitoe, 1999). The silicified zones in the Mazra'eh Shadi mining districts are surrounded by argillic alteration halos. Both Barnes (1979) and Krauskopf (1979) showed that there was evidence to suggest that chloride complexes were more stable than sulphide complexes at higher temperatures (above $350^{\circ} \mathrm{C}$ ). The greater stability of chloride complexes at higher temperatures with respect to sulphide complexing is corroborated by the modelling studies of $\mathrm{Ag} / \mathrm{Au}$ ratios in hydrothermal solutions by Cole \& Drummond (1986). They found that $\mathrm{AuCl}^{-2}$ complexes predominate at temperatures higher than $250^{\circ} \mathrm{C}$ and low to moderate $\mathrm{pH}$, thus favouring high $\mathrm{Ag} / \mathrm{Au}$ ratios in the solution. At temperatures below $250^{\circ} \mathrm{C}$ sulphide complexes with more Au than Ag predominate, leading to lower $\mathrm{Ag} / \mathrm{Au}$ ratios in the solution.

According to Barnes (1979), deposition of metals from sulphide complexes depends on the following factors: 1) Pressure release and boiling; 2) Oxidation, decreasing the sulphide contents and $\mathrm{pH}$. Thus, oxidation within and above the zone of boiling results in the formation of sulphuric acid $\left(\mathrm{H}_{2} \mathrm{SO}_{4}\right)$, a pH decrease and acid leaching (argillic alteration). During this process, electrons are released that reduce the $\mathrm{Au}^{+}$to its neutral state $\mathrm{Au}^{0}$ causing its precipitation as a native metal. Other important mechanisms for the precipitation of the dissolved constituents are chemical reactions between the solutions and wall rocks. Mineralisation of Au in Mazra'eh Shadi deposits due to pyrite precipitation, boiling of hydrothermal fluids and decreasing of $\mathrm{pH}$.

\section{Conclusions}

The main results of the present study are the following:

- Statistical analyses confirm a significant correlation between $\mathrm{Au}, \mathrm{Ag}, \mathrm{Pb}, \mathrm{Zn}$, and a strong correlation between $\mathrm{Pb}-\mathrm{Ag}$ and $\mathrm{Pb}-\mathrm{Zn}$ in $\mathrm{Au}$-bearing quartz veins. 
- It is possible that another pluton intruded within the first pluton in the region and caused the irregular shape and $\mathrm{Ag}^{*} \mathrm{~Pb}^{*} \mathrm{Zn} / \mathrm{Cu}^{*} \mathrm{Bi}^{*} \mathrm{Co}$ ratio against depth.

- The longitudinal sections show clearly that $\mathrm{Cu}$, $\mathrm{Pb}, \mathrm{Zn}$ and Mo occur at lower elevations, whereas $\mathrm{Au}$ and $\mathrm{Ag}$ seem to occur at shallower levels. This observation contradicts the typical zoning pattern caused by boiling in epithermal veins.

- Correlation between Au-Ag and closest association this element in dendrogram with $\mathrm{Fe}$ and $\mathrm{S}$ in Au-bearing quartz veins are due deposition this element as a result of pyrite precipitation in temperatures $<350^{\circ} \mathrm{C}$.

- Mineralisation of Au and Ag in the Mazra'eh Shadi deposit is due to pyrite precipitation, boiling of hydrothermal fluids and decreasing of $\mathrm{pH}$.

\section{Acknowledgements}

The authors are grateful to the research committee of Lorestan University for supporting this project and to anonymous reviewers for commenting on an earlier typescript and supplying valuable suggestions.

\section{References}

Barnes, H.L., 1979. Solubilities of ore minerals. [In:] H.L, Barnes (Ed). Geochemistry of hydrothermal ore deposits, $2^{\text {nd }}$ ed. John Wiley \& Sons, New York. pp 404-410.

Bodnar, R.J., 1993. Revised equation and table for determining the freezing point depression of $\mathrm{H}_{2} \mathrm{O}-\mathrm{NaCl}$ solutions. Geochimca et Cosmochimica Acta 57, 683-684.

Browne, P.R.L., 1978. Hydrothermal alteration in active geothermal fields. Annual Reviews of Earth and Planetary Science 6, 229-250.

Browne, P.R.L. \& Ellis., A.J., 1970. The Ohaki-Broadlands hydrothermal area, New Zealand: Mineralogy and related geochemistry. American Journal of Science 269, 97-131.

Buchanan, L.J., 1981. Precious Metal Deposits Associated with Volcanic Environments in the Southwest: In Relations of Tectonics to Ore Deposits in the Southern Cordillera. Geological Society of Arizona, Digest 14, 237-262.

Cole, D.R. \& Drummond, S.E., 1986. The effect of transport and boiling on $\mathrm{Ag} / \mathrm{Au}$ ratios in hydrothermal solutions: a preliminary assessment and possible implications for the formation of epithermal precious metal ore deposits. Journal of Geochemical Exploration 25, 45-79.

Davis, C., 1986. Statistics and Data Analysis in Geology. John Wiley \& Sons, New York. 640 pp.

Dreier, J.E., 2005. The Environment of vein Formation and Ore Deposition in the Purisima-Colon Vein System, Pachuca Real del Monte District, Hidalgo, Mexico. Economic Geology 100, 1325 - 1347.
Grancea, L., Bailey, L., Leroy, J., Banks, D., Marcoux, E., Milési, J.P., Cuney, M., André, A.S., Istvan, D. \& Fabre, C., 2002. Fluid evolution in the Baia Mare epithermal gold/polymetallic district, Inner Carpathians, Romani. Mineral Deposita 37, 630-647.

Hedenquist, J.W., Arribas, A.R. \& Gonzalez-Urien, E., 2000. Exploration for epithermal gold deposits. Reviews in Economic Geology 13, 245-277.

Henley, R.W., Brown, K.L., 1985. A practical guide to the thermodynamics of geothermal fluids and hydrothermal ore deposits. Reviews in Economic Geology 2, 25-44.

Howarth, R.I., 1993. Statistics and Data Analysis in Geochimical Prospecting. [In:] G.J.S, Govett (Ed). Handbook of Exploration Geochemistry. Elsever, Amsterdam. 2, 44-75.

Krauskopf, K.B., 1979. Introduction to geochemistry. $2^{\text {nd }}$ ed. McGraw-Hill Kogakushu, New York. 617pp.

McLemore, V.T., 2008. Geochemistry and statistical analyses of epithermal veins at the Carlisle and Center mines, Steeple Rock District. New Mexico, USA. Arizona Geological Society Digest 22, 485-496.

Nabavi, M., 1976. An Introduction to the Geology of Iran. Geological Society of Iran, Teheran. (in Persian). 109 pp.

Nie, N.H., Hull, C.H. \& Jenkins, J.G., Steinbrenner, K. \& Bent, P.H., 1975. Statistical package for the Social Sciences. McGraw-Hill Book Co, New York, 675 pp.

Pokrovski, G.S., Borisova, Yu.A. \& Harrichoury, J.C., 2008. The effect of sulfur on vapor- liquid fractionation of metals in hydrothermal systems. Earth and Planetary Science Letters 266, 345-362.

Radmard, K., Zamanian, H., Hosseinzadeh, M.R. \& Ahmadi Khalaj, A., 2017. Geochemistry and hydrothermal evolution of the Mazraeh Shadi-Hizehjan precious and base metal deposit, northeastern Tabriz, Iran. Journal of Mineralogy and Geochemistry 194-3, 227-250.

Rassi, R. \& Afzal, P., 2015. Correlation between Au Lithogeochemical Anomalies and Fault-density using Geostatistical and Fractal Modeling in Sharaf Abad-Hizehjan Area, NW Iran. Universal Journal of Geoscience 3, 51-58.

Sillitoe, R.H., 1999. Styles of high-sulfidation gold, silver and copper mineralization in porphyry and epithermal environments. [In:] Weber, G. (Ed.): Pacrim '99 Congress, Bali, Indonesia. Proceedings. Australasian Institute of Mining and Metallurgy, Parkville, 29-44.

Sillitoe, R.H. \& Hedenquist, J.W., 2003. Linkage between volcanotectonic settings, ore-fluid compositions, and epithermal precious- metal deposits. Society of Economic Geologist, Special Publication 10, 315-343.

Simmons, S.F. \& Browne, P.R.L., 2000. Hydrothermal minerals and precious metals in the Broadlands-Ohaaki geothermal system: Implications for understanding low-sulfidation epithermal environments. Economic Geology 95, 971-999.

Tuysuz, N. \& Yaylali, G., 2005. Geostatistics. Karadenis Technical University Publication 220, 400 pp.

Manuscript received: 5 June 2017 Revision accepted: 15 September 2017 